Does improving sleep lead to better mental health?

\title{
Improving Sleep Quality Leads to Better Mental Health: A Meta-Analysis of Randomised Controlled Trials
}

Alexander J. Scott ${ }^{*}$, Thomas L. Webb ${ }^{3}$, Marrissa Martyn-St James ${ }^{2}$, Georgina Rowse ${ }^{4}$, and Scott Weich ${ }^{2}$.

${ }^{1}$ Keele University, School of Psychology, Keele, UK.

${ }^{2}$ School of Health and Related Research (ScHARR), The University of Sheffield, UK.

${ }^{3}$ Department of Psychology, The University of Sheffield, UK.

${ }^{4}$ Clinical Psychology Unit, Department of Psychology, The University of Sheffield, UK.

\section{Manuscript accepted at Sleep Medicine Reviews}

Funding: This research was funded by the National Institute for Health Research (NIHR) under its Research for Patient Benefit (RfPB) Programme (Grant Reference Number PB-PG0817-20027). The views expressed are those of the author(s) and not necessarily those of the NIHR or the Department of Health and Social Care.

*Corresponding author: Dr Alexander Scott, School of Psychology, Dorothy Hodgkin Building, Keele University, Staffordshire, ST5 5BG. a.scott@keele.ac.uk. 
Does improving sleep lead to better mental health?

\section{Summary}

The extent to which sleep is causally related to mental health is unclear. One way to test the causal link is to evaluate the extent to which interventions that improve sleep quality also improve mental health. We conducted a meta-analysis of randomised controlled trials that reported the effects of an intervention that improved sleep on composite mental health, as well as on seven specific mental health difficulties. 65 trials comprising 72 interventions and $N=8,608$ participants were included. Improving sleep led to a significant medium-sized effect on composite mental health $(g+=-0.53)$, depression $(g+=-0.63)$, anxiety $(g+=-0.51)$, and rumination $\left(g^{+}=-0.49\right)$, as well as significant small-to-medium sized effects on stress $\left(g^{+}=-\right.$ 0.42), and finally small significant effects on positive psychosis symptoms $(g+=-0.26)$. We also found a dose response relationship, in that greater improvements in sleep quality led to greater improvements in mental health. Our findings suggest that sleep is causally related to the experience of mental health difficulties. Future research might consider how interventions that improve sleep could be incorporated into mental health services, as well as the mechanisms of action that explain how sleep exerts an effect on mental health.

Keywords: Sleep; insomnia; CBTi; mental health; meta-analysis; causal inference; depression, anxiety; stress; psychosis. 


\section{Does Improving Sleep Lead to Better Mental Health? A Meta-Analysis of Randomised Controlled Trials}

Problems sleeping are common. A review of several hundred epidemiological studies (1) concluded that nearly one-third of the general population experience symptoms of insomnia (defined as difficulties falling asleep and/or staying asleep), between $4 \%$ and $26 \%$ experience excessive sleepiness, and between $2 \%$ and $4 \%$ experience obstructive sleep apnoea. Additionally, a recent study of over 2000 participants reported that the prevalence of 'general sleep disturbances' was 32\% (2) and Chattu et al. concluded on the basis of a large systematic review of the evidence that public and health professionals need to be more aware of the adverse effects of poor sleep (3). Mental health problems are also common, with around $17 \%$ of adults experiencing mental health difficulties of varying severities (4), and evidence from large nationally representative studies suggesting that that mental health difficulties are on the increase (5). Sleep and mental health are, therefore, global public health challenges in their own right, with each having substantive impacts on both individuals and society $(3,6,7)$. However, problems sleeping and mental health difficulties are also intrinsically linked $(8,9)$. It was previously assumed that mental health difficulties led to problems sleeping $(10,11)$; however, the reverse may also be true (12), such that poor sleep contributes to the onset, recurrence, and maintenance of mental health difficulties (13-17). Therefore, the extent to which there is a causal relation between (poor) sleep and (worse) mental health and the possibility that interventions designed to improve sleep might be able to reduce mental health difficulties warrants investigation.

\section{Evidence on the relationship between sleep and mental health}

The association between sleep and mental health is well documented $(9,13,18-23)$. For example, people with insomnia are 10 and 17 times more likely than those without insomnia to 
Does improving sleep lead to better mental health?

experience clinically significant levels of depression and anxiety, respectively (24). Furthermore, a meta-analysis of 21 longitudinal studies reported that people with insomnia at baseline had a two-fold risk of developing depression at follow-up compared with people who did not experience insomnia (13). Although research most commonly studies the associations between insomnia and depression and anxiety, there is also evidence that problems sleeping are associated with a variety of mental health difficulties. For example, poor sleep has also been associated with post-traumatic stress (25), eating disorders (26), and psychosis spectrum experiences such as delusions and hallucinations $(23,27)$. Studies have also found that specific sleep disorders, such as sleep apnoea (28), circadian rhythm disruption (29), restless leg syndrome (30), excessive daytime sleepiness and narcolepsy $(31,32)$, sleepwalking (33), and nightmares (34) are all more prevalent in those experiencing mental health difficulties.

Unfortunately, most research on the association between sleep and mental health is observational in design. While informative, inferring causation from such studies is difficult. For example, cross-sectional designs tell us that variables are associated in some way, but they cannot say whether one variable precedes the other in a causal chain (35). Longitudinal designs provide stronger evidence, but are prone to residual confounding (36-38) and other forms of bias that limit causal inference (39-43). The best evidence is provided by studies that randomly allocate participants to experimental and control conditions to minimise the effects of potential confounds $(44,45)$. Therefore, to establish whether sleeping problems are causally associated with mental health difficulties, it is necessary to experimentally manipulate sleep to see whether changes in sleep lead to changes in mental health over time (i.e., the interventionist approach to causation, 46). 
Does improving sleep lead to better mental health?

Many RCTs have examined the effect of interventions designed to improve sleep (typically cognitive behavioural therapy for insomnia, CBTi), on mental health (typically depression and anxiety). There have also been attempts to meta-analyse some of these RCTs and quantify their effects on mental health outcomes (47-50). However, even these meta-analyses do not permit robust conclusions as to the causal impact of sleep on mental health outcomes for several reasons. First, previous reviews have included studies that did not successfully manipulate sleep (i.e., the intervention did not improve sleep relative to controls). It is not possible to conclude whether sleep is causally linked to mental health if the experimental manipulation of sleep is unsuccessful (51). Indeed, these studies simply tell us that it can sometimes be difficult to improve sleep in the first place. Second, reviews have tended to examine the effect of interventions targeting sleep on mental health at the first post-intervention time point. This is problematic for two reasons; (i) there is no temporal lag between the measurement of sleep and measurement of mental health (a key tenet of causal inference); and (ii) effects are limited to the short-term where they are likely to be strongest. Third, the focus of previous reviews has been limited to depression and anxiety only, and typically limited to CBTi interventions. Therefore, the effect of improving sleep on other mental health outcomes, using different approaches to intervention, is limited. Finally, to date there has been no or limited attempts to investigate variables that influence - or moderate - the impact of interventions that improve sleep on mental health. It is crucial that the impact of such variables is systematically examined to understand whether the effect of improving sleep on mental health differs across populations, settings, and study designs. 


\section{The present review: An interventionist approach to causation}

The present review sought to address these issues to provide an accurate and robust estimate of the effect of changes in sleep quality (i.e., as a result of an intervention) on changes in mental health. To test this empirically, we identified randomised controlled trials that successfully manipulated sleep in an intervention group relative to controls, and then measured mental health at a later follow-up point. We did not limit the scope of interventions to CBTi, or the measures of mental health to solely depression and/or anxiety. Instead, we included any intervention designed to improve sleep that produced a statistically significant effect on sleep quality relative to controls and examined the effect of that improvement in sleep on any subsequent mental health outcome. To better isolate the effect of improved sleep on mental health, we excluded interventions that included specific elements targeting mental health (e.g., CBT elements for depression). Given the (potentially) high degree of heterogeneity between studies that this approach might create, we examined the effect of different study characteristics and outcomes using moderation analyses. Our primary hypothesis is that interventions that significantly improve sleep will lead to significantly improved mental health at follow-up.

\section{Method}

\section{Eligibility criteria}

To be included in the present review, studies needed to (i) be a randomized controlled trial that tested an intervention designed to improve sleep; (ii) produce a statistically significant effect on sleep quality when compared to a control group or an alternative treatment, (iii) report a measure of mental health subsequent to the measure of sleep quality, (iv) report sufficient data to compute an effect size representing the impact of the intervention on both sleep quality and mental health, (v) be written in English, or translatable using available resources. In order to 
Does improving sleep lead to better mental health?

reliably and validly assess the independent contribution of changes in sleep on mental health outcomes among adult populations, studies were excluded if (i) the intervention contained elements that specifically target a mental health problem in addition to elements that target sleep; or (ii) recruited children and young people (i.e., < 18 years of age).

\section{Search strategy}

First, we searched MEDLINE (1946 to present), Embase (1974 to present), PsycINFO (1967 to present), and The Cochrane Library (1898 to present) using the Cochrane Highly Sensitive Search Strategy (i.e., HSSS, 52) to identify RCTs that included terms relating to sleep quality and/or sleep disorders, and mental health (see Table 1 for a list of the search terms and Supplementary Material 1 for an example search strategy). Second, the reference lists of extant reviews of the relationship between sleep and mental health were searched for any potential articles. Third, a search for any unpublished or ongoing studies was conducted by searching online databases including White Rose Online, The National Research Register, WHO approved clinical trial databases (e.g., ISRCTN), and PROSPERO. Searches were originally conducted in May 2019 and then updated in February 2021.

\section{*INSERT TABLE 1 HERE*}

\section{Data management and study selection}

We followed PRISMA guidelines (53) when selecting studies. The first phase of screening removed duplicate records and records that were clearly ineligible based on the title and / or abstract. The second phase of screening cross-referenced full-text versions of articles against the inclusion criteria, with eligible records included in the present review, and ineligible records excluded along with reasons for exclusion. Records were screened by two members of the review team, and a sub-sample of $10 \%$ of each reviewer's records were second checked by 
Does improving sleep lead to better mental health?

the other reviewer, with almost perfect agreement between the reviewers (kappa $=1.00$ and 0.99).

\section{Data extraction}

Data was extracted from included studies using a standardized form and an accompanying manual detailing each variable for extraction. In addition to extracting statistical data to compute effect sizes, data pertaining to source characteristics of included studies (e.g., publication status, year, impact factor), characteristics of the sample (e.g., age, type of mental health problem), the study (e.g., the nature of the comparison group, length of follow-up), and the intervention (e.g., intervention type, mode of delivery) was also extracted.

\section{Outcomes and prioritization}

\section{Measuring improvements in sleep}

The concept of 'improved sleep' is multifaceted and can mean different things to different people (54-56). Consequently, one challenge for the proposed review was to ensure that included studies assessed a similar notion of improved sleep so that they could be meaningfully combined using a single metric. Therefore, we specified that primary studies reported a measure that reflected the overall quality of sleep experienced by participants. The concept of sleep quality can also be subjective (54); however, broadly speaking, sleep quality consists of Sleep continuity (e.g., sleep onset, sleep maintenance, and number of awakenings) and daytime impact (e.g., the extent to which the person feels refreshed on waking and throughout the day, see 54,57). We used the following hierarchy to decide which outcome measure(s) to use to estimate an effect size (in descending order of prioritization); (i) self-report measures of global sleep quality (e.g., the Pittsburgh Sleep Quality Index); (ii) outcomes specific to a given sleep disorder that assess sleep continuity and impact on daily life (e.g., the Insomnia Severity Index); 
Does improving sleep lead to better mental health?

and (iii) individual components of self-reported sleep continuity aggregated to form a single composite effect size (e.g., the average effect of intervention on sleep onset latency (SoL) and wake after sleep onset (WASO)).

\section{Measuring mental health}

We examined the effect of improving sleep on (i) composite mental health (which included all mental health outcomes reported across studies, see Table 2 for outcomes), and (ii) specific mental health difficulties in isolation (e.g., depression separately from other mental health outcomes). We computed the between-group effect of improving sleep on each mental health outcome reported by the study at the furthest follow-up point available. This strategy provides a stringent test of the effect of improving sleep on mental health outcomes in the sense that any changes need to have been maintained over time. In line with previous reviews (58), these effect sizes were then averaged to form a 'composite' measure of mental health. As with the measures of sleep quality, we prioritized self-report measures of mental health rather than observer-rated measures, as arguably it is the subjective experience of mental health problems that is most important (59).

\section{Risk of bias}

Risk of bias was assessed using the risk of bias assessment criteria developed by the Cochrane Collaboration (60). RCTs were classified as being at overall risk of bias according to three of the six domains - (i) allocation concealment, (ii) blinding of outcome assessment and (iii) completeness of outcome data (attrition). RCTs judged as being at low risk of bias for all three domains were judged at overall low risk of bias. RCTs judged as being at high risk for any of the three domains were judged as overall high risk of bias. RCTs judged as a mix of low and 
Does improving sleep lead to better mental health?

unclear risk on these three domains, or all unclear were judged as unclear with respect to risk of bias.

\section{Estimating effect sizes}

Hedges $g$ and the associated standard error were estimated using the means and standard deviations reported by each of the primary studies. Where means and standard deviations were not reported, effect sizes were estimated by converting relevant summary statistics into Hedges g. Where studies reported multiple outcome measures for the same/similar constructs (e.g., several measures of depression), effect sizes were computed for each outcome and then metaanalysed in their own right to form one overall effect.

\section{Meta-analytic approach}

All analyses were conducted in R (61), using the 'esc' (62), 'meta' (63), 'metafor' (64), 'dmetar' (65), and 'robvis' (66) packages. The pooled, sample-weighted, average effect size was computed using a random effects model as effect sizes between studies are likely to vary considerably (67). Following Cohen's recommendations (68), $g=0.20$ was taken to represent a 'small' effect size, $g=0.50$ a 'medium' effect size and $g=0.80$ a 'large' effect size. The $I^{2}$ statistic was used to assess heterogeneity of effect sizes across the included studies and was interpreted according to the classifications suggested by Higgins et al. (69), where $I^{2}=25 \%$ indicates low heterogeneity, $I^{2}=50 \%$ indicates moderate heterogeneity, and $I^{2}=75 \%$ indicates high heterogeneity. Publication bias was assessed via visual inspection of a funnel plot and Egger's test (70). Additionally, Orwin's formula (71) was used to determine the fail-safe $n$. Finally, outliers were defined as any effect size for which the confidence intervals did not overlap with the confidence interval of the pooled effect (72). We conducted a sensitivity 
Does improving sleep lead to better mental health?

analysis examining the effect of outliers for each outcome by rerunning the analysis with any outlying effect sizes removed.

\section{Subgroup analyses}

Moderation analysis was conducted to identify variables that were associated with the effect of improving sleep on mental health outcomes. A minimum of 3 studies representing each moderator level category was required in order to conduct moderation analysis. For categorical variables, the analysis was based on a mixed effects model, in that the pooling of effect sizes within each moderator level was based on a random effects model, while the comparison of effect sizes between levels was based on a fixed effects model. The $Q$ statistic was then used to assess whether effect sizes were significantly different between moderator levels. For continuous variables, sample-weighted meta-regression was used to investigate the impact of the moderator on mental health effect sizes.

\section{Data availability statement}

All data and analysis code are freely available on the Open Science Framework under a creative commons 4.0 license (for access, see 73 ).

\section{Study Selection}

\section{Results}

Figure 1 shows the flow of records through the review. Systemic searches of the published and grey literature retrieved a total of 21,733 records, which was reduced to 15,139 after duplicates were removed. Of these records, 14,687 (97\%) were excluded in the first stage of screening, leaving 452 full-text records to be screened. Of these records, 387 (86\%) were crossreferenced against the review eligibility criteria and excluded (see Figure 1 for a breakdown of 
Does improving sleep lead to better mental health?

reasons and Supplementary Materials 2 for a list of the studies excluded at this stage), leaving 65 records for inclusion in the meta-analysis.

\section{*INSERT FIGURE 1 HERE*}

\section{Study characteristics}

Table 2 describes key characteristics of the included studies. The 65 studies provided 72 comparisons between an intervention that successfully improved sleep quality ys. a control group.

\section{Participants}

A total of $N=8,608$ participants took part across the 72 interventions. 38 of the comparisons (53\%) included participants with a comorbid physical or mental health problem, while $31(43 \%)$ reported no comorbid health problems, and $3(4 \%)$ reported insufficient detail to make a judgement. Of the 38 comparisons including participants with comorbid health problems, $18(47 \%)$ reported mental health diagnoses, and $20(53 \%)$ had physical health problems.

\section{Outcome measures}

The majority of comparisons $(61,85 \%)$ reported a measure of depression, but $33(46 \%)$ reported a measure of anxiety, $6(8 \%)$ reported a measure of stress, $5(7 \%)$ reported measures of psychosis spectrum experiences (e.g., total, positive, and negative symptoms), 9 (13\%) reported a measure of general mood, $2(3 \%)$ reported post-traumatic stress disorder outcomes, $2(3 \%)$ reported measures of suicidal ideation, $4(6 \%)$ reported rumination outcomes, and $1(2 \%)$ reported a measure of psychological burnout.

\section{Interventions and comparisons}

Most interventions were multi-component CBTi (53, 74\%), but interventions also involved acupuncture $(7,10 \%)$, pharmacological treatments $(2,3 \%)$, sleep hygiene alone $(2$, 
Does improving sleep lead to better mental health?

$3 \%)$, sleep restriction alone (2, 3\%), Tai Chi (2, 3\%), CBT for nightmares $(1,2 \%)$, herbal remedies $(1,2 \%)$, walking $(1,2 \%)$, and yoga $(1,2 \%)$. Interventions were most often compared against an active control group (34, 47\%), but were also compared to waitlist control groups (25, $35 \%)$, and groups receiving treatment as usual $(13,18 \%)$. On average participants' mental health was followed-up 20.5 weeks post-intervention (median = 12 weeks post-intervention), with the earliest follow-up being 4-weeks post-intervention, and the furthest follow-up 156-weeks (three years) post intervention.

Manipulation check: Did sleep quality improve significantly in the intervention group relative to controls?

Before we examined the effect of improving sleep quality on subsequent mental health, we confirmed that studies included in the review successfully improved sleep quality. The interventions had large and statistically significant effects on sleep quality at the earliest followup point reported $\left(g_{+}=-1.07,95 \% \mathrm{CI}=-1.26\right.$ to $\left.-0.88, p<0.001\right)$, although heterogeneity between studies was substantial $\left(I^{2}=79 \%, Q=331.93, p<0.001\right)$. After twelve outlying effect sizes were removed, the effect of the interventions on sleep quality remained large and statistically significant $\left(g_{+}=-0.97,95 \% \mathrm{CI}=-1.07\right.$ to $\left.-0.88, p<0.001\right)$, and heterogeneity was reduced to moderate levels $\left(I^{2}=43 \%, Q=102.32, p<0.001\right)$. These findings suggest that the primary studies included in the present review successfully manipulated sleep quality, even after accounting for outliers.

\section{What effect do improvements in sleep quality have on mental health?}

Table 3 presents the effect of improving sleep quality on composite mental health outcomes, and on measures of depression, anxiety, stress, psychosis spectrum experiences, suicidal ideation, PTSD, rumination, and burnout. 
Does improving sleep lead to better mental health?

\section{*INSERT TABLE 3 HERE*}

\section{Composite mental health}

On average, the 72 interventions that successfully improved sleep quality had a statistically significant, medium-sized effect on subsequent composite mental health outcomes, $\left(g_{+}=-0.53,95 \% \mathrm{CI}=-0.68\right.$ to $\left.-0.38, p<0.001\right)$; however, there was substantial heterogeneity between the effect sizes, $\left(I^{2}=76 \%, Q=291.94, p<0.001\right)$. After re-running the analysis with eleven outlying effect sizes removed, the effect of improving sleep on composite mental health outcomes was small-to-medium sized but still statistically significant, $\left(g_{+}=-0.42,95 \% \mathrm{CI}=-\right.$ 0.49 to $-0.34, p<0.001)$ and now relatively homogeneous $\left(I^{2}=20 \%, Q=75.24, p=0.0888\right)$. See Figure 2 for a forest plot.

\section{*INSERT FIGURE 2 HERE*}

\section{Depression}

Interventions that successfully improved sleep quality had a statistically significant, medium-sized effect on depression across 61 comparisons, $\left(g_{+}=-0.63,95 \% \mathrm{CI}=-0.83\right.$ to -0.43 , $p<0.001)$; however, once again, there was substantial heterogeneity, $\left(I^{2}=81 \%, Q=322.09, p<\right.$ 0.001). After re-running the analysis with nine outlying effect sizes removed, the effect of improving sleep on depression remained medium-sized, $\left(g_{+}=-0.47,95 \% \mathrm{CI}=-0.57\right.$ to $-0.37, p<$ 0.001), with moderate heterogeneity, $\left(I^{2}=32 \%, Q=74.86, p=0.0164\right)$. See Figure 3 for a forest plot.

\section{*INSERT FIGURE 3 HERE*}

\section{Anxiety}

Interventions that successfully improved sleep quality had a statistically significant, small-to-medium sized effect on anxiety across 35 comparisons, $\left(g_{+}=-0.50,95 \% \mathrm{CI}=-0.76\right.$ to - 
Does improving sleep lead to better mental health?

$0.24, p<0.001)$, with substantial levels of heterogeneity, $\left(I^{2}=82 \%, Q=187.02, p<0.001\right)$. After re-running the analysis with four outlying effect sizes removed, the effect improving sleep on anxiety outcomes was small-to-medium sized, but still statistically significant, $\left(g_{+}=-0.38\right.$, $95 \% \mathrm{CI}=-0.49$ to $-0.27, p<0.001)$, with lower levels of heterogeneity, $\left(I^{2}=43 \%, Q=52.49, p\right.$ $=0.0067)$. See Figure 4 for a forest plot.

*INSERT FIGURE 4 HERE*

\section{Stress}

Interventions that successfully improved sleep quality had a statistically significant, small-to-medium sized effect on stress $\left(g_{+}=-0.42,95 \% \mathrm{CI}=-0.79\right.$ to $\left.-0.05, p=0.033\right)$, across 6 comparisons. There were moderate levels of heterogeneity $\left(I^{2}=55 \%, Q=11.05, p=0.05\right)$, but there were no outlying effect sizes. See Figure 5 for a forest plot.

\section{*INSERT FIGURE 5 HERE*}

\section{Psychosis spectrum experiences}

Interventions that successfully improved sleep quality had a small effect on total symptoms as indicated by the PANSS $\left(g_{+}=-0.17,95 \% \mathrm{CI}=-0.53\right.$ to $\left.0.19, p=0.18\right)$ across 3 comparisons, with zero heterogeneity $\left(I^{2}=0 \%, Q=0.41, p=0.813\right)$. Interventions that successfully improved sleep quality had a small effect on positive symptoms $\left(g_{+}=-0.26,95 \%\right.$ CI $=-0.43$ to $-0.08, p=0.014)$ across 5 comparisons, with zero heterogeneity $\left(I^{2}=0 \%, Q=1.71, p\right.$ $=0.788$ ). Finally, interventions that successfully improved sleep quality had a small effect on negative symptoms $\left(g_{+}=-0.28,95 \% \mathrm{CI}=-3.22\right.$ to $\left.2.65, p=0.436\right)$ across $k=2$ comparisons, with zero heterogeneity $\left(I^{2}=0 \%, Q=1, p=0.318\right)$. See Figure 6 for a forest plot. 
Does improving sleep lead to better mental health?

\section{Suicidal ideation}

Interventions that successfully improved sleep quality had a small, adverse effect on suicidal ideation $\left(g_{+}=0.10,95 \% \mathrm{CI}=-3.74\right.$ to $\left.3.94, p=0.804\right)$ across 2 comparisons. There were low levels of heterogeneity $\left(I^{2}=20 \%, Q=1.25, p=0.263\right)$ and no outlying effect sizes. See Figure 5 for a forest plot.

\section{Post-traumatic stress disorder (PTSD)}

Interventions that successfully improved sleep quality had a medium-to-large effect on $\operatorname{PTSD}\left(g_{+}=-0.72,95 \% \mathrm{CI}=-2.90\right.$ to $\left.1.46, p=0.149\right)$ across 2 comparisons, with zero heterogeneity $\left(I^{2}=0 \%, Q=0.59, p=0.442\right)$. See Figure 5 for a forest plot.

\section{Rumination}

Interventions that successfully improved sleep quality had a statistically significant, medium sized effect on rumination $\left(g_{+}=-0.49,95 \% \mathrm{CI}=-0.93\right.$ to $\left.-0.04, p=0.041\right)$ across 4 comparisons, with moderate heterogeneity $\left(I^{2}=36 \%, Q=4.65, p=0.1991\right)$. See Figure 5 for a forest plot.

\section{Burnout}

Only 1 study reported the effect of improving sleep on burnout finding almost zero effect $(g=-0.03, \mathrm{CI}=-0.58$ to $0.52, p=0.917)$.

\section{Moderators of the effect of improving sleep quality on composite mental health outcomes}

Table 4 presents the findings of analyses evaluating categorical moderators of the effect of improving sleep quality on composite mental health outcomes and Table 5 presents analyses evaluating continuous moderators using meta-regression. Studies that found significant effects of the intervention on sleep quality reported larger effects on subsequent composite mental health, $(g=-0.53,95 \% \mathrm{CI}=-0.68$ to $-0.38, p<.0 .001)$, than studies that did not find a significant effect 
Does improving sleep lead to better mental health?

of the intervention on sleep quality, $(g=-0.12,95 \% \mathrm{CI}=-0.24$ to $0.01, p=0.0522)$, a difference that was statistically significant, $(Q=17.59, p<.001)$. This finding strengthens the notion that improvements in sleep are behind improvements in mental health. The effect of improving sleep on mental health was larger in studies with shorter follow-up periods, (i.e., $<6$ months, $g+=-$ 0.60), than in studies with longer follow-ups, (i.e., $\geq 6$ months, $g+=-0.18, Q=10.75, p<0.01$ ). Furthermore, interventions that were delivered face-to-face by a clinician or therapist were associated with significantly larger effects on mental health, $(g+=-0.63)$, than those that were self-administered by participants, $(g+=-0.34, Q=4.50, p<0.05)$. Finally, there was significant variation in the size of the effect between countries $(Q=53.69, p<.001)$. No other statistically significant categorical moderator effects were found. Regarding continuous moderators, metaregression revealed a statistically significant dose-response effect for the association between the effect of interventions on sleep quality and the effect on subsequent mental health outcomes $(B=$ $0.77,95 \% \mathrm{CI}=0.52$ to $1.02, p<.001)$, suggesting that greater improvements in sleep led to greater improvements in mental health. No other continuous variables significantly moderated the effect of improving sleep on mental health.

\section{Post-hoc moderation analysis}

Is the smaller effect of improving sleep on mental health at longer follow-ups

\section{associated with smaller effects on sleep quality?}

We conducted further (unplanned) post-hoc analysis to investigate whether the smaller effect of improving sleep on mental health at longer follow-ups was accompanied by a reduction in the improvements to sleep quality. Studies reporting the effect of the intervention at shorter follow-ups reported larger improvements in sleep quality, $(g=-1.03,95 \% \mathrm{CI}=-1.27$ to $-0.78, p$ $<.0 .001)$, than those reporting longer follow-ups $(g=-0.44,95 \% \mathrm{CI}=-0.62$ to $-0.27, p<$ 
Does improving sleep lead to better mental health?

$.0 .001)$, a difference that was statistically significant, $(Q=14.38, p<.001)$. This suggests that the smaller effect of improving sleep on mental health at longer follow-ups might be driven by a smaller effect of the interventions on sleep quality at longer follow-ups.

\title{
Can some of the effect of improved mental health be explained by CBTi modules that
}

\section{target processes associated with mental health?}

Finally, although the present review excluded interventions that specifically and directly targeted mental health, some CBTi protocols include modules that might target similar processes associated with some mental health difficulties (rumination around sleep, catastrophizing over the effect of poor sleep etc.). Therefore, we compared CBTi interventions with modules that could target processes associated with mental health vs. interventions that did not include these modules (e.g., sleep restriction alone, sleep hygiene alone, herbal tea, and pharmacological intervention). There were no significant differences in the effect of improved sleep quality on mental health between CBTi interventions including modules addressing processes associated with mental health $(g=-0.44,95 \% \mathrm{CI}=-0.59$ to $-0.29, p<.0 .001)$, relative to those that did not $(g=-0.48,95 \% \mathrm{CI}=-0.65$ to $-0.32, p<.0 .001, Q=2.51, p=0.285)$. This finding suggests that it is the beneficial effect of improved sleep quality that confers improvements in mental health rather than the inclusion of modules that target processes associated with mental health commonly seen in CBTi protocols.

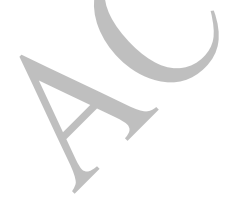

\author{
*INSERT TABLE 4 HERE* \\ *INSERT TABLE 5 HERE*
}

\section{Risk of bias assessments}

Figure 7 summarizes the weighted assessment of risk of bias. Individual risk of bias judgements for included studies are presented in Supplementary Material 3. Ten studies (15\%) 
Does improving sleep lead to better mental health?

were judged as having low risk of bias, 29 studies (45\%) were judged as high risk of bias, and 26 studies $(40 \%)$ were judged as unclear. The methodological quality of the included studies was not associated with the effect of improving sleep on composite mental health outcomes, $Q=$ $0.72, p=0.395$.

\section{*INSERT FIGURE 7 HERE*}

\section{Publication bias}

A funnel plot of the effect of improving sleep quality on composite outcomes revealed asymmetry in the effect sizes (Egger's regression $=-1.09,95 \% \mathrm{CI}=-1.91$ to $-0.28, p<0.05$, see Figure 8). Duval and Tweedie's (74) trim and fill procedure was therefore used to address the asymmetry. Ten studies were imputed resulting in a statistically significant, small-to-medium sized adjusted effect of improving sleep on composite mental health outcomes $\left(g_{+}=-0.35,95 \%\right.$ $\mathrm{CI}=-0.55$ to $-0.16, p<0.001)$. Orwin's failsafe $n$ test suggested that an additional 4,101 comparisons producing null effects would be needed to reduce the average effect of improving sleep on composite outcomes to zero. Taken together these results suggest that the effect of improving sleep on composite mental health is robust to possible publication bias.

\section{*INSERT FIGURE 8 HERE*}

\section{Discussion}

The present review used meta-analysis to synthesize the effect of 72 interventions that improved sleep quality relative to a control condition on subsequent mental health. The findings revealed that improving sleep quality had, on average, a medium-sized effect on mental health, including clear evidence that improving sleep reduced depression, anxiety, and stress. A dearth of primary studies of other mental health difficulties (e.g., psychosis spectrum experiences, suicidal ideation, PTSD, rumination, and burnout) mean that it is premature to draw definitive 
conclusions in these areas. It was also notable that we found a dose-response relationship between improvements in sleep quality and subsequent mental health, such that greater improvements in sleep led to greater improvements in mental health. Although there was some evidence of publication bias, the effects remained robust to correction. Taken together, the findings suggest that improving sleep leads to better mental health, therefore providing strong evidence that sleep plays a causal role in the experience of mental health difficulties.

\section{Sleep as a transdiagnostic treatment target}

The present findings support the idea that targeting sleep promotes mental health across a range of populations and experiences. The effect of improving sleep quality on composite mental health was medium-sized and statistically significant, regardless of the presence of physical and/or mental health comorbidities. This finding is particularly important given the healthcare challenges associated with multimorbidity (75) and mental and physical health problems often co-occur (76-79), something that appears to be increasing (80). Consequently, it is important that the benefits of improving sleep on mental health occur even in the presence of comorbid health complaints, as was reported in the present research. Improving sleep has also been shown to improve aspects of physical health, including fatigue (81), chronic pain $(82,83)$, and overall health related quality of life (84) and could reduce the cost of healthcare. For example, offering a digital CBTi intervention (Sleepio) to primary care patients was associated with an average saving of $£ 70.44$ per intervention user (85), and cost savings following sleep intervention have also been specifically reported in people with comorbid mental health difficulties such as depression (86).

Another finding to suggest that targeting sleep could promote mental health across a range of populations and experiences, is that we found no difference in the effect of improving 
Does improving sleep lead to better mental health?

sleep quality on mental health between those with clinically defined mental health difficulties and those with non-clinical experiences or between those recruited from clinical vs. community settings, with both groups receiving significant benefits of improved sleep on mental health. This suggests that improving sleep could prove helpful across a range of mental health severities, thus broadening the possible impact of sleep interventions within healthcare services. Finally, there is growing evidence that sleep disturbances predict the development of mental health difficulties in the future. For example, shorter and more variable sleep has been shown to be longitudinally associated with more severe hallucinations and delusional ideation in those at high-risk of psychosis (87). The present research found that improving sleep has a significant beneficial impact on future mental health in those with non-clinical experiences, raising the possibility that delivering interventions that improve sleep early might limit the risk of developing (or exacerbating) substantive mental health difficulties. Indeed, less severe mild-to-moderate presentations of mental health difficulties can develop over time into more severe mental health diagnoses $(88,89)$, therefore improving sleep might be one tool that can be used in combination with others to limit the risk of transition.

\section{Strengths and limitations}

The present review has several strengths. First, it provides a comprehensive and up-todate search of RCTs examining the effect of improving sleep on a variety of subsequent mental health outcomes. Indeed, with $65 \mathrm{RCTs}$ and $N=8,608$ participants, the present review is one of the largest studies of the effect of improving sleep on mental health to date. Second, the review was specifically designed to test the causal association between sleep and mental health (i.e., RCTs only, successful sleep improvement required, temporal lag between measures etc.). To our knowledge, the review is the first to adopt this approach in the field of sleep and mental health, 
Does improving sleep lead to better mental health?

although the general approach has been used in other fields (90). Finally, we provide an analysis of possible moderators of the effect of improving sleep on mental health, identifying several key moderators of the effect.

However, there are limitations that must be considered when interpreting the findings. First, relatively few studies examined the effect of improving sleep over the long term. Those that did report longer follow-ups generally found smaller effects (although still statistically significant), most likely due to the diminishing effects of interventions on sleep quality over time (91). Consequently, it is important that interventions targeting sleep quality as a route to improving mental health seek to maintain their beneficial effects. Second, there were few primary studies for some of the outcomes included in this review. Consequently, in lieu of more studies reporting these outcomes, the inferences that we can make for mental health outcomes other than depression and anxiety are more limited. Third, although the intention of the present review was to include a broad range of sleep disturbances, most of the analyses are based on CBT interventions for insomnia. This might be due to the relationship between insomnia and mental health being the one that is historically most studied. However, it may be that our focus on sleep quality precluded some studies that do not focus on insomnia from inclusion. For example, different sleep disorders have different conceptualisations of improvement that might not include sleep quality. For example, the timing of sleep is particularly important in circadian rhythm disorders and daytime sleepiness is a key outcome in sleep apnoea research. Future research might consider examining the effect of improving specific sleep disorders on mental health by conceptualising improvements using sleep disorder specific outcomes. 
Does improving sleep lead to better mental health?

\section{Future directions}

The present review highlighted several areas for future research in terms of both research and theory, and the implementation of findings in practice. First, given that mental health was measured on average around 20.5 weeks post-intervention in the primary studies, and that the effect of improving sleep on mental health significantly reduced over time, future research should examine the effect of improving sleep on mental health over the longer term. Second, although not uncommon, the majority of RCTs included in the present review were at high risk, or unclear risk or bias. Consequently, in addition to studying the effect of improving sleep over the longer term, on a range of mental health difficulties beyond depression and anxiety, we need more research at lower risk of methodological bias.

Finally, although the present research provides evidence for a causal association between sleep and mental health, it is less clear how sleep effects mental health. One potential mechanism is whether and how people regulate their emotions (e.g., in response to negative events). Indeed, evidence suggests that poor sleep can amplify the adverse effect of negative life events $(92,93)$, dull the beneficial impact of positive events (94), and is associated with more frequent use of emotion regulation strategies that might be detrimental to good mental health (95). By extension, although we are unaware of RCTs testing the effect of improved sleep on emotion regulation, changes in sleep are prospectively associated with changes in aspects of emotion regulation $(96,97)$, while experimentally induced sleep deprivation is adversely linked to poorer emotion regulation $(96,97)$. Contemporary perspectives on emotion regulation (e.g., the action control perspective), draw on research on how people regulate their behaviour, to propose that regulating emotions involves three tasks, (i) identifying the need to regulate, (ii) deciding whether and how to regulate, and (iii) enacting a regulation strategy (98). We propose that poor sleep quality has 
Does improving sleep lead to better mental health?

the potential to adversely affect anyone (or all) of the three tasks involved in effectively regulating emotions, which might go some way toward explaining the relationship between poor sleep and mental health. Therefore, we would recommend that future research includes measures of aspects of emotion regulation (e.g., the Difficulties in Emotion Regulation Scale, 99) within experimental and longitudinal designs to elucidate possible mechanisms by which improvements in sleep benefit mental health.

In terms of practice and implementation, evidence on the effect of sleep on mental health also supports calls for routine screening and treatment of problems with sleep. Both the Royal Society for Public Health (RSPH) and the Mental Health Foundation (MHF) recommend that primary health care training should include awareness of, and skills in assessing, sleep problems $(100,101)$. Despite this and a growing body of evidence, there has been little progress to date (102). This may reflect under-appreciation of the importance of sleep (103) and lack of training and skills in assessing and managing sleep problems (104-108), as well as limited time and resources $(103,109)$. Therefore, a profitable next step might be to explore barriers and facilitators to assessing sleep and delivering effective interventions in specific care settings, from both the patient and clinician perspective. Indeed, the present review also highlighted a dearth of trials that tested the effect of improving sleep on mental health outcomes in 'real world' settings (e.g., within existing clinical and community health services). Although some researchers are taking important steps in this area (110-112), there is a clear need for more trials of interventions in clinical services so that the effectiveness and implementation of such interventions in routine care can be better understood. 
Does improving sleep lead to better mental health?

\section{Conclusions}

Taken together, the present research supports the view that sleep is causally related to the experience of mental health difficulties, and therefore that sleep represents a viable treatment target that can confer significant benefits to mental health, as it has been found to do for physical health. We found that improving sleep was associated with better mental health regardless of the severity of mental health difficulty (i.e., clinical vs. non-clinical) or the presence of comorbid health conditions. Poor sleep is almost ubiquitous within mental health services $(102,108,113,114)$, is causally related to the experience of mental health difficulties, and represents a potential treatment target $(105,115,116)$. Consequently, equipping health professionals with greater knowledge and resources to support sleep is an essential next step. Future research should consider how interventions that improve sleep could be better incorporated into routine mental health care, as well as the possible mechanisms of action that might explain how sleep exerts its effects on mental health. 


\section{Practice points}

- Sleep is causally related to the experience of mental health difficulties and represents a viable transdiagnostic treatment target for those experiencing mental health difficulties.

- Improving sleep has beneficial effects on the experience of mental health difficulties, regardless of the severity of those difficulties, or the presence of comorbid health conditions.

- Healthcare professionals aiming to improve mental health (particularly depression, anxiety, and stress) should consider interventions designed to improve sleep, particularly cognitive behavioral therapy for insomnia where the evidence base is strongest.

\section{Research agenda}

To fully harness the effect of improved sleep on mental health, it is important that future research:

1. Explores the barriers and possible solutions to incorporating interventions that improve sleep into mental health care services.

2. Tests the effect of improving sleep on mental health outcomes beyond depression and anxiety, and over the long term, using designs at low risk of methodological bias.

3. Investigates the possible mechanisms of action that might explain how sleep exerts its effects on the experience of mental health difficulties. 
Does improving sleep lead to better mental health?

\section{References}

1. Ohayon MM. Epidemiological overview of sleep disorders in the general population. Sleep Med Res. 2011;2(1):1-9.

2. Kerkhof GA. Epidemiology of sleep and sleep disorders in The Netherlands. Sleep Med. 2017;30:229-39.

3. Chattu VK, Manzar MD, Kumary S, Burman D, Spence DW, Pandi-Perumal SR. The global problem of insufficient sleep and its serious public health implications. In: Healthcare. Multidisciplinary Digital Publishing Institute; 2019.

4. Bebbington PE, McManus S. Revisiting the one in four: the prevalence of psychiatric disorder in the population of England 2000-2014. Br J Psychiatry. 2020;216(1):55-7.

5. Twenge JM, Cooper AB, Joiner TE, Duffy ME, Binau SG. Age, period, and cohort trends in mood disorder indicators and suicide-related outcomes in a nationally representative dataset, 2005-2017. J Abnorm Psychol. 2019;128(3):185.

6. Hale L, Troxel W, Buysse DJ. Sleep Health: An Opportunity for Public Health to Address Health Equity. Annu Rev Public Health. 2020;41.

7. Robotham D. Sleep as a public health concern: insomnia and mental health. J Public Ment Health. 2011;

8. Ford DE, Kamerow DB. Epidemiologic study of sleep disturbances and psychiatric disorders: an opportunity for prevention? Jama. 1989;262(11):1479-84.

9. Baglioni C, Nanovska S, Regen W, Spiegelhalder K, Feige B, Nissen C, et al. Sleep and mental disorders: A meta-analysis of polysomnographic research. Psychol Bull. 2016;142(9):969.

10. Stepanski EJ, Rybarczyk B. Emerging research on the treatment and etiology of secondary or comorbid insomnia. Sleep Med Rev. 2006;10(1):7-18.

11. McCrae CS, Lichstein KL. Secondary insomnia: diagnostic challenges and intervention opportunities. Sleep Med Rev. 2001 Feb;5(1):47-61.

12. *Alyaro PK, Roberts RM, Harris JK. A systematic review assessing bidirectionality between sleep disturbances, anxiety, and depression. Sleep. 2013;36(7):1059-68.

13. *Baglioni C, Battagliese G, Feige B, Spiegelhalder K, Nissen C, Voderholzer U, et al. Insomnia as a predictor of depression: a meta-analytic evaluation of longitudinal epidemiological studies. J Affect Disord. 2011;135(1-3):10-9.

14. Gregory AM, Rijsdijk FV, Lau JY, Dahl RE, Eley TC. The direction of longitudinal associations between sleep problems and depression symptoms: a study of twins aged 8 and 10 years. Sleep. 2009;32(2):189-99. 
Does improving sleep lead to better mental health?

15. *Harvey AG. Insomnia: Symptom or diagnosis? Clin Psychol Rev. 2001 Oct;21(7):103759.

16. Soehner AM, Kaplan KA, Harvey AG. Insomnia comorbid to severe psychiatric illness. Sleep Med Clin. 2013;8(3):361-71.

17. Freeman D, Stahl D, McManus S, Meltzer H, Brugha T, Wiles N, et al. Insomnia, worry, anxiety and depression as predictors of the occurrence and persistence of paranoid thinking. Soc Psychiatry Psychiatr Epidemiol. 2012 Aug;47(8):1195-203.

18. Benca RM, Obermeyer WH, Thisted RA, Gillin JC. Sleep and psychiatric disorders: a meta-analysis. Arch Gen Psychiatry. 1992;49(8):651-68.

19. Breslau N, Roth T, Rosenthal L, Andreski P. Sleep disturbance and psychiatric disorders: a longitudinal epidemiological study of young adults. Biol Psychiatry. 1996;39(6):411-8.

20. Chan M-S, Chung K-F, Yung K-P, Yeung W-F. Sleep in schizophrenia: a systematic review and meta-analysis of polysomnographic findings in case-control studies. Sleep Med Rev. 2017;32:69-84.

21. Kobayashi I, Boarts JM, Delahanty DL. Polysomnographically measured sleep abnormalities in PTSD: a meta-analytic review. Psychophysiology. 2007;44(4):660-9.

22. Nota JA, Sharkey KM, Coles ME. Sleep, arousal, and circadian rhythms in adults with obsessive-compulsive disorder: A meta-analysis. Neurosci Biobehav Rev. 2015;51:100-7.

23. *Reeve S, Sheaves B, Freeman D. The role of sleep dysfunction in the occurrence of delusions and hallucinations: a systematic review. Clin Psychol Rev. 2015;42:96-115.

24. Taylor DJ, Lichstein KL, Durrence HH, Reidel BW, Bush AJ. Epidemiology of insomnia, depression, and anxiety. Sleep. 2005;28(11):1457-64.

25. Harvey AG, Jones C, Schmidt DA. Sleep and posttraumatic stress disorder: a review. Clin Psychol Rev. 2003;23(3):377-407.

26. Lauer CJ, Krieg J-C. Sleep in eating disorders. Sleep Med Rev. 2004;8(2):109-18.

27. Scott AJ, Rowse G, Webb TL. A structural equation model of the relationship between insomnia, negative affect, and paranoid thinking. PLOS ONE. 2017;12(10):e0186233.

28. Sharafkhaneh A, Giray N, Richardson P, Young T, Hirshkowitz M. Association of psychiatric disorders and sleep apnea in a large cohort. Sleep. 2005;28(11):1405-11.

29. Wulff K, Gatti S, Wettstein JG, Foster RG. Sleep and circadian rhythm disruption in psychiatric and neurodegenerative disease. Nat Rev Neurosci. 2010;11(8):589-99.

30. Picchietti D, Winkelman JW. Restless legs syndrome, periodic limb movements in sleep, and depression. Sleep. 2005;28(7):891-8. 
Does improving sleep lead to better mental health?

31. Dodel R, Peter H, Spottke A, Noelker C, Althaus A, Siebert U, et al. Health-related quality of life in patients with narcolepsy. Sleep Med. 2007;8(7-8):733-41.

32. Plante DT. Hypersomnia in mood disorders: a rapidly changing landscape. Curr Sleep Med Rep. 2015;1(2):122-30.

33. Ohayon MM, Guilleminault C, Priest RG. Night terrors, sleepwalking, and confusional arousals in the general population: their frequency and relationship to other sleep and mental disorders. J Clin Psychiatry. 1999;60(4):268-76.

34. Hasler BP, Germain A. Correlates and treatments of nightmares in adults. Sleep Med Clin. 2009;4(4):507-17.

35. Brewer MB, Crano WD. Research design and issues of validity. Handb Res Methods Soc Personal Psychol. 2000;3-16.

36. Achen $\mathrm{CH}$. Let's put garbage-can regressions and garbage-can probits where they belong. Confl Manag Peace Sci. 2005;22(4):327-39.

37. Spector PE, Brannick MT. Methodological urban legends: The misuse of statistical control variables. Organ Res Methods. 2011;14(2):287-305.

38. Zapf D, Dormann C, Frese M. Longitudinal studies in organizational stress research: a review of the literature with reference to methodological issues. J Occup Health Psychol. 1996;1(2):145.

39. Elwert F, Winship C. Endogenous selection bias: The problem of conditioning on a collider variable. Annu Rev Sociol. 2014;40:31-53.

40. Morgan SL, Winship C. Counterfactuals and causal inference. Cambridge University Press; 2015.

41. Pearl J, Glymour M, Jewell NP. Causal inference in statistics: A primer. John Wiley \& Sons; 2016.

42. Rohrer JM. Thinking clearly about correlations and causation: Graphical causal models for observational data. Adv Methods Pract Psychol Sci. 2018;1(1):27-42.

43. Duckworth AL, Tsukayama E, May H. Establishing causality using longitudinal hierarchical linear modeling: An illustration predicting achievement from self-control. Soc Psychol Personal Sci. 2010;1(4):311-7.

44. Woodward J. Making things happen: A theory of causal explanation. Oxford university press; 2005.

45. Cartwright N. Hunting causes and using them: Approaches in philosophy and economics. Cambridge University Press; 2007. 
Does improving sleep lead to better mental health?

46. *Campbell J. An interventionist approach to causation in psychology. Causal Learn Psychol Philos Comput. 2007;58-66.

47. *Gebara MA, Siripong N, DiNapoli EA, Maree RD, Germain A, Reynolds CF, et al. Effect of insomnia treatments on depression: A systematic review and meta-analysis. Depress Anxiety. 2018;35(8):717-31.

48. Ho FY-Y, Chan CS, Lo W-Y, Leung JC-Y. The Effect of Self-Help Cognitive Behavioral Therapy for Insomnia on Depressive Symptoms: An Updated Meta-Analysis of Randomized Controlled Trials. J Affect Disord. 2020;

49. Belleville G, Cousineau H, Levrier K, St-Pierre-Delorme M-È. Meta-analytic review of the impact of cognitive-behavior therapy for insomnia on concomitant anxiety. Clin Psychol Rev. 2011;31(4):638-52.

50. Gee B, Orchard F, Clarke E, Joy A, Clarke T, Reynolds S. The effect of nonpharmacological sleep interventions on depression symptoms: A meta-analysis of randomised controlled trials. Sleep Med Rev. 2019;43:118-28.

51. Fedak KM, Bernal A, Capshaw ZA, Gross S. Applying the Bradford Hill criteria in the 21st century: how data integration has changed causal inference in molecular epidemiology. Emerg Themes Epidemiol. 2015;12(14).

52. Glanville JM, Lefebvre C, Miles JN, Camosso-Stefinovic J. How to identify randomized controlled trials in MEDLINE: ten years on. J Med Libr Assoc. 2006;94(2):130.

53. Moher D, Liberati A, Tetzlaff J, Altman DG. Preferred reporting items for systematic reviews and meta-analyses: the PRISMA statement. BMJ. 2009 Jul 21;339:b2535.

54. Harvey AG, Stinson K, Whitaker KL, Moskovitz D, Virk H. The subjective meaning of sleep quality: a comparison of individuals with and without insomnia. Sleep. 2008;31(3):383-93.

55. Crivello A, Barsocchi P, Girolami M, Palumbo F. The meaning of sleep quality: a survey of available technologies. IEEE Access. 2019;7:167374-90.

56. Krystal AD, Edinger JD. Measuring sleep quality. Sleep Med. 2008;9:S10-7.

57. Libman E, Fichten C, Creti L, Conrod K, Tran D-L, Grad R, et al. Refreshing sleep and sleep continuity determine perceived sleep quality. Sleep Disord. 2016;2016.

58. Wu JQ, Appleman ER, Salazar RD, Ong JC. Cognitive behavioral therapy for insomnia comorbid with psychiatric and medical conditions: a meta-analysis. JAMA Intern Med. 2015;175(9):1461-72.

59. Strauss J. Subjectivity and severe psychiatric disorders. Schizophr Bull. 2011;37(1):8-13. 
Does improving sleep lead to better mental health?

60. Higgins JP, Altman DG, Sterne JA. Chapter 8: Assessing risk of bias in included studies. Cochrane Handbook for Systematic Reviews of Interventions Version 5.1. 0 [updated March 2011]. Cochrane Collab. 2011;

61. Team RC. R: A language and environment for statistical computing. 2013;

62. Lüdecke, Daniel. esc: Effect Size Computation for Meta Analysis [Internet]. 2019. Available from: https://CRAN.R-project.org/package=esc

63. Balduzzi S, Rücker G, Schwarzer G. How to perform a meta-analysis with R: a practical tutorial. Evid Based Ment Health. 2019 Nov;22(4):153-60.

64. Viechtbauer W. Conducting meta-analyses in R with the metafor package. J Stat Softw. 2010;36(3):1-48.

65. Harrer M, Cuijpers P, Furukawa T, Ebert DD. dmetar: Companion R Package For The Guide “Doing Meta-Analysis in R". R package version 0.0. 9000. 2019.

66. McGuinness LA, Higgins JPT. Risk-of-bias VISualization (robvis): An R package and Shiny web app for visualizing risk-of-bias assessments. Res Synth Methods [Internet]. 2020 Apr 26 [cited 2020 May 21];n/a(n/a). Available from: https://doi.org/10.1002/jrsm.1411

67. Borenstein M, Hedges LV, Higgins JP, Rothstein HR. A basic introduction to fixed-effect and random-effects models for meta-analysis. Res Synth Methods. 2010;1(2):97-111.

68. Cohen J. A power primer. Psychol Bull. 1992;112(1):155.

69. Higgins JP, Thompson SG, Deeks JJ, Altman DG. Measuring inconsistency in metaanalyses. Bmj. 2003;327(7414):557-60.

70. Egger M, Smith GD, Schneider M, Minder C. Bias in meta-analysis detected by a simple, graphical test. Bmj. 1997;315(7109):629-34.

71. Orwin RG. A fail-safe N for effect size in meta-analysis. J Educ Stat. 1983;8(2):157-9.

72. Harrer M, Cuijpers P, Furukawa TA, Ebert DD. Doing meta-analysis in R: A hands-on guide. Prot Lab Erlangen. 2019;

73. Scott A, Webb TL, Martyn-St James M, Rowse G, Weich S. Supplementary materials for "Improving Sleep Quality Leads to Better Mental Health: A Meta-Analysis of Randomised Controlled Trials". [Internet]. 2021. Available from: osf.io/6edjv

74. Duval S, Tweedie R. Trim and fill: a simple funnel-plot-based method of testing and adjusting for publication bias in meta-analysis. Biometrics. 2000;56(2):455-63.

75. Nguyen H, Manolova G, Daskalopoulou C, Vitoratou S, Prince M, Prina AM. Prevalence of multimorbidity in community settings: A systematic review and meta-analysis of observational studies. J Comorbidity. 2019;9:2235042X19870934. 
Does improving sleep lead to better mental health?

76. Leucht S, Burkard T, Henderson JH, Sartorius N, Maj M. Physical illness and schizophrenia: a review of the evidence. Cambridge University Press; 2007.

77. Sareen J, Cox BJ, Stein MB, Afifi TO, Fleet C, Asmundson GJ. Physical and mental comorbidity, disability, and suicidal behavior associated with posttraumatic stress disorder in a large community sample. Psychosom Med. 2007;69(3):242-8.

78. Jones DR, Macias C, Barreira PJ, Fisher WH, Hargreaves WA, Harding CM. Prevalence, severity, and co-occurrence of chronic physical health problems of persons with serious mental illness. Psychiatr Serv. 2004;55(11):1250-7.

79. El-Gabalawy R, Mackenzie CS, Shooshtari S, Sareen J. Comorbid physical health conditions and anxiety disorders: a population-based exploration of prevalence and health outcomes among older adults. Gen Hosp Psychiatry. 2011;33(6):556-64.

80. Sartorious N. Comorbidity of mental and physical diseases: a main challenge for medicine of the 21st century. Shanghai Arch Psychiatry. 2013;25(2):68.

81. Espie CA, Fleming L, Cassidy J, Samuel L, Taylor LM, White CA, et al. Randomized controlled clinical effectiveness trial of cognitive behavior therapy compared with treatment as usual for persistent insomnia in patients with cancer. J Clin Oncol. 2008;26(28):4651-8.

82. Selvanathan J, Pham C, Nagappa M, Peng PW, Englesakis M, Espie CA, et al. Cognitive Behavioral Therapy for Insomnia in Patients with Chronic Pain-A Systematic Review and Meta-Analysis of Randomized Controlled Trials. Sleep Med Rev. 2021;101460.

83. Vitiello MV, Rybarczyk B, Von Korff M, Stepanski EJ. Cognitive behavioral therapy for insomnia improves sleep and decreases pain in older adults with co-morbid insomnia and osteoarthritis. J Clin Sleep Med. 2009;5(04):355-62.

84. Kyle SD, Morgan K, Espie CA. Insomnia and health-related quality of life. Sleep Med Rev. 2010;14(1):69-82.

85. Sampson CJ, Bell E, Cole A, Miller CB, Rose J. Digital cognitive behavioural therapy for insomnia and primary care costs in England: an interrupted time series analysis. medRxiv. 2021;

86. Watanabe N. Cost-effectiveness of brief behavioral therapy for insomnia comorbid with depression: analysis of a randomized controlled trial. Psychosom Med. 2014;76(3):A-90.

87. Reeve S, Nickless A, Sheaves B, Hodgekins J, Stewart SLK, Gumley A, et al. Sleep duration and psychotic experiences in patients at risk of psychosis: a secondary analysis of the EDIE-2 trial. Schizophr Res. 2019;204:326-33.

88. Van Os J, Linscott RJ, Myin-Germeys I, Delespaul P, Krabbendam L. A systematic review and meta-analysis of the psychosis continuum: evidence for a psychosis pronenesspersistence-impairment model of psychotic disorder. Psychol Med. 2009;39(2):179-95. 
Does improving sleep lead to better mental health?

89. Keyes CL. The mental health continuum: From languishing to flourishing in life. J Health Soc Behav. 2002;207-22.

90. Webb TL, Sheeran P. Does changing behavioral intentions engender behavior change? A meta-analysis of the experimental evidence. Psychol Bull. 2006;132(2):249.

91. van der Zweerde T, Bisdounis L, Kyle SD, Lancee J, van Straten A. Cognitive behavioral therapy for insomnia: a meta-analysis of long-term effects in controlled studies. Sleep Med Rev. 2019;48:101208.

92. O'Leary K, Bylsma LM, Rottenberg J. Why might poor sleep quality lead to depression? A role for emotion regulation. Cogn Emot. 2017;31(8):1698-706.

93. Gujar N, Yoo S-S, Hu P, Walker MP. Sleep Deprivation Amplifies Reactivity of Brain Reward Networks, Biasing the Appraisal of Positive Emotional Experiences. J Neurosci. 2011;31(12):4466-74.

94. Zohar D, Tzischinsky O, Epstein R, Lavie P. The effects of sleep loss on medical residents' emotional reactions to work events: a cognitive-energy model. Sleep. 2005;28(1):47-54.

95. Zhang J, Lau EYY, Hsiao JH. Using emotion regulation strategies after sleep deprivation: ERP and behavioral findings. Cogn Affect Behav Neurosci. 2019;19(2):283-95.

96. Vandekerckhove M, Wang Y. Emotion, emotion regulation and sleep: An intimate relationship. Aims Neurosci. 2018;5(1):1.

97. Palmer CA, Alfano CA. Sleep and emotion regulation: an organizing, integrative review. Sleep Med Rev. 2017;31:6-16.

98. Webb TL, Schweiger Gallo I, Miles E, Gollwitzer PM, Sheeran P. Effective regulation of affect: An action control perspective on emotion regulation. Eur Rev Soc Psychol. 2012;23(1):143-86.

99. Gratz KL, Roemer L. Multidimensional Assessment of Emotion Regulation and Dysregulation: Development, Factor Structure, and Initial Validation of the Difficulties in Emotion Regulation Scale. J Psychopathol Behav Assess. 2004 Mar 1;26(1):41-54.

100. *Mental Health Foundation. Sleep Matters: The Impact of Sleep on Health and Wellbeing. Mental Health Foundation London, UK; 2011.

101. *Royal Society for Public Health. Waking up to the health benefits of sleep [Internet]. Royal Society for Public Health; 2016. Available from: https://www.rsph.org.uk/static/uploaded/a565b58a-67d1-4491-ab9112ca414f7ee4.pdf

102. *Freeman D, Sheaves B, Waite F, Harvey AG, Harrison PJ. Sleep disturbance and psychiatric disorders. Lancet Psychiatry. 2020 Jul 1;7(7):628-37. 
Does improving sleep lead to better mental health?

103. Meaklim H, Jackson ML, Bartlett D, Saini B, Falloon K, Junge M, et al. Sleep education for healthcare providers: Addressing deficient sleep in Australia and New Zealand. Sleep Health. 2020;

104. Cross E, Ellis J, Draghi-Lorenz R. The Role of Sleep and Sleep Disorders in the Therapeutic Encounter: An Ipa Study of Counselling Psychologists in the Uk. Sleep. 2009;32:A390-A390.

105. Davy Z, Middlemass J, Siriwardena AN. Patients' and clinicians' experiences and perceptions of the primary care management of insomnia: qualitative study. Health Expect. 2015;18(5):1371-83.

106. Ellis J. Sleep and psychology curriculum. Oxf Handb Sleep Sleep Disord. 2012;289-300.

107. Meltzer LJ, Phillips C, Mindell JA. Clinical psychology training in sleep and sleep disorders. J Clin Psychol. 2009;65(3):305-18.

108. O'Sullivan M, Rahim M, Hall C. The prevalence and management of poor sleep quality in a secondary care mental health population. J Clin Sleep Med. 2015;11(2):111-6.

109. Romiszewski S, May FEK, Homan EJ, Norris B, Miller MA, Zeman A. Medical student education in sleep and its disorders is still meagre 20 years on: A cross-sectional survey of UK undergraduate medical education. J Sleep Res. 2020;e12980.

110. Stott R, Pimm J, Emsley R, Miller CB, Espie CA. Does adjunctive digital CBT for insomnia improve clinical outcomes in an improving access to psychological therapies service? Behav Res Ther. 2021;144:103922.

111. Kraepelien M, Forsell E, Blom K. Large-scale implementation of insomnia treatment in routine psychiatric care: patient characteristics and insomnia-depression comorbidity. $\mathrm{J}$ Sleep Res. 2021;e13448.

112. Kyle SD, Madigan C, Begum N, Abel L, Armstrong S, Aveyard P, et al. Primary care treatment of insomnia: study protocol for a pragmatic, multicentre, randomised controlled trial comparing nurse-delivered sleep restriction therapy to sleep hygiene (the HABIT trial). Vol. 10, BMJ Open. 2020. p. e036248.

113. Arroll B, Fernando A, Falloon K, Goodyear-Smith F, Samaranayake C, Warman G. Prevalence of causes of insomnia in primary care: a cross-sectional study. Br J Gen Pract. 2012;62(595):e99-103.

114. Haynes PL, Parthasarathy S, Kersh B, Bootzin RR. Examination of insomnia and insomnia treatment in psychiatric inpatients. Int J Ment Health Nurs. 2011;20(2):130-6.

115. Waite F, Evans N, Myers E, Startup H, Lister R, Harvey AG, et al. The patient experience of sleep problems and their treatment in the context of current delusions and hallucinations. Psychol Psychother Theory Res Pract. 2016;89(2):181-93. 
Does improving sleep lead to better mental health?

116. Faulkner S, Bee P. Perspectives on sleep, sleep problems, and their treatment, in people with serious mental illnesses: a systematic review. PloS One. 2016;11(9):e0163486.

117. Alessi C, Martin JL, Fiorentino L, Fung CH, Dzierzewski JM, Rodriguez Tapia JC, et al. Cognitive Behavioral Therapy for Insomnia in Older Veterans Using Nonclinician Sleep Coaches: Randomized Controlled Trial. J Am Geriatr Soc. 2016;64(9):1830-8.

118. Ashworth DK, Sletten TL, Junge M, Simpson K, Clarke D, Cunnington D, et al. A randomized controlled trial of cognitive behavioral therapy for insomnia: an effective treatment for comorbid insomnia and depression. J Couns Psychol. 2015;62(2):115-23.

119. Behrendt D, Ebert DD, Spiegelhalder K, Lehr D. Efficacy of a Self-Help Web-Based Recovery Training in Improving Sleep in Workers: Randomized Controlled Trial in the General Working Population. Vol. 22, Journal of Medical Internet Research. 2020. p. e13346.

120. Bergdahl L, Broman JE, Berman AH, Haglund K, von Knorring L, Markstrom A. Auricular Acupuncture and Cognitive Behavioural Therapy for Insomnia: A Randomised Controlled Study. Sleep Disord Print. 2016;2016:7057282.

121. Blom K, Jernelov S, Ruck C, Lindefors N, Kaldo V. Three-year follow-up comparing cognitive behavioral therapy for depression to cognitive behavioral therapy for insomnia, for patients with both diagnoses. Vol. 40, Sleep. 2017.

122. Cape J, Leibowitz J, Whittington C, Espie CA, Pilling S. Group cognitive behavioural treatment for insomnia in primary care: a randomized controlled trial. Psychol Med. 2016;46(5):1015-25.

123. Casault L, Savard J, Ivers H, Savard MH. A randomized-controlled trial of an early minimal cognitive-behavioural therapy for insomnia comorbid with cancer. Behav Res Ther. 2015;67:45-54.

124. Chang SM, Chen CH. Effects of an intervention with drinking chamomile tea on sleep quality and depression in sleep disturbed postnatal women: a randomized controlled trial. J Adv Nurs. 2016;72(2):306-15.

125. Chang YL, Chiou AF, Cheng SM, Lin KC. Tailored educational supportive care programme on sleep quality and psychological distress in patients with heart failure: A randomised controlled trial. Int J Nurs Stud. 2016;61:219-29.

126. Chao LL, Kanady JC, Crocker N, Straus LD, Hlavin J, Metzler TJ, et al. Cognitive behavioral therapy for insomnia in veterans with gulf war illness: Results from a randomized controlled trial. Life Sciences. 2021. p. 119147.

127. Chen KM, Chen MH, Chao HC, Hung HM, Lin HS, Li CH. Sleep quality, depression state, and health status of older adults after silver yoga exercises: cluster randomized trial. Int $\mathbf{J}$ Nurs Stud. 2009;46(2):154-63. 
Does improving sleep lead to better mental health?

128. Chen IH, Yeh TP, Yeh YC, Chi MJ, Chen MW, Chou KR, et al. Effects of Acupressure on Sleep Quality and Psychological Distress in Nursing Home Residents: a Randomized Controlled Trial. Vol. 20, Journal of the American Medical Directors Association. 2019. p. 822-829.

129. Cheng P, Kalmbach DA, Tallent G, Joseph CL, Espie CA, Drake CL. Depression prevention via digital cognitive behavioral therapy for insomnia: a randomized controlled trial. Vol. 42, Sleep. 2019. p. 09.

130. *Christensen H, Batterham PJ, Gosling JA, Ritterband LM, Griffiths KM, Thorndike FP, et al. Effectiveness of an online insomnia program (SHUTi) for prevention of depressive episodes (the GoodNight Study): a randomised controlled trial. Lancet Psychiatry. 2016;3(4):333-41.

131. Chung KF, Yeung WF, Yu BY, Leung FC, Zhang SP, Zhang ZJ, et al. Acupuncture with or without combined auricular acupuncture for insomnia: a randomised, waitlist-controlled trial. Acupunct Med. 2018;36(1):2-13.

132. Currie SR. Cognitive-behavioural treatment of insomnia secondary to chronic pain. Diss Abstr Int Sect B Sci Eng. 2000 Feb;60(8-B):4212.

133. Edinger JD, Wohlgemuth WK, Krystal AD, Rice JR. Behavioral insomnia therapy for fibromyalgia patients: a randomized clinical trial. Arch Intern Med. 2005;165(21):2527-35.

134. Espie CA, Kyle SD, Miller CB, Ong J, Hames P, Fleming L. Attribution, cognition and psychopathology in persistent insomnia disorder: outcome and mediation analysis from a randomized placebo-controlled trial of online cognitive behavioural therapy. Sleep Med. 2014;15(8):913-7.

135. Espie CA, Emsley R, Kyle SD, Gordon C, Drake CL, Siriwardena AN, et al. Effect of Digital Cognitive Behavioral Therapy for Insomnia on Health, Psychological Well-being, and Sleep-Related Quality of Life: a Randomized Clinical Trial. Vol. 76, JAMA psychiatry. 2019. p. 21-30.

136. Falloon K, Elley CR, Fernando A 3rd, Lee AC, Arroll B. Simplified sleep restriction for insomnia in general practice: a randomised controlled trial. Br J Gen Pract. 2015;65(637):e508-515.

137. Felder JN, Epel ES, Neuhaus J, Krystal AD, Prather AA. Efficacy of Digital Cognitive Behavioral Therapy for the Treatment of Insomnia Symptoms Among Pregnant Women: A Randomized Clinical Trial. Vol. 77, JAMA Psychiatry. 2020. p. 484-92.

138. Freeman D, Waite F, Startup H, Myers E, Lister R, McInerney J, et al. Efficacy of cognitive behavioural therapy for sleep improvement in patients with persistent delusions and hallucinations (BEST): a prospective, assessor-blind, randomised controlled pilot trial. Lancet Psychiatry. 2015;2(11):975-83. 
Does improving sleep lead to better mental health?

139. Freeman D, Sheaves B, Goodwin GM, Yu L-M, Nickless A, Harrison PJ, et al. The effects of improving sleep on mental health (OASIS): a randomised controlled trial with mediation analysis. Lancet Psychiatry. 2017;4(10):749-58.

140. Garland SN, Carlson LE, Stephens AJ, Antle MC, Samuels C, Campbell TS. Mindfulnessbased stress reduction compared with cognitive behavioral therapy for the treatment of insomnia comorbid with cancer: a randomized, partially blinded, noninferiority trial. J Clin Oncol. 2014;32(5):449-57.

141. Garland SN, Xie SX, DuHamel K, Bao T, Li Q, Barg FK, et al. Acupuncture Versus Cognitive Behavioral Therapy for Insomnia in Cancer Survivors: A Randomized Clinical Trial. Vol. 111, Journal of the National Cancer Institute. 2019. p. 1323-31.

142. Germain A, Richardson R, Moul DE, Mammen O, Haas G, Forman SD, et al.Placebocontrolled comparison of prazosin and cognitive-behavioral treatments for sleep disturbances in US Military Veterans. J Psychosom Res. 2012;72(2):89-96.

143. Glozier N, Christensen H, Griffiths KM, Hickie IB, Naismith SL, Biddle D, et al. Adjunctive Internet-delivered cognitive behavioural therapy for insomnia in men with depression: A randomised controlled trial. Aust N Z J Psychiatry. 2019 Apr;53(4):350-60.

144. Ham OK, Lee BG, Choi E, Choi SJ. Efficacy of Cognitive Behavioral Treatment for Insomnia: A Randomized Controlled Trial. Vol. 42, Western Journal of Nursing Research. 2020. p. 1104-12.

145. Ho FY, Chung KF, Yeung WF, Ng TH, Cheng SK. Weekly brief phone support in self-help cognitive behavioral therapy for insomnia disorder: Relevance to adherence and efficacy. Behav Res Ther. 2014;63:147-56.

146. Irwin MR, Olmstead R, Carrillo C, Sadeghi N, Breen EC, Witarama T, et al. Cognitive behavioral therapy vs. Tai Chi for late life insomnia and inflammatory risk: a randomized controlled comparative efficacy trial. Sleep. 2014;37(9):1543-52.

147. Jansson-Frojmark M, Linton SJ, Flink IK, Granberg S, Danermark B, Norell-Clarke A. Cognitive-behavioral therapy for insomnia co-morbid with hearing impairment: a randomized controlled trial. J Clin Psychol Med Settings. 2012;19(2):224-34.

148. Jernelov S, Lekander M, Blom K, Rydh S, Ljotsson B, Axelsson J, et al. Efficacy of a behavioral self-help treatment with or without therapist guidance for co-morbid and primary insomnia--a randomized controlled trial. BMC Psychiatry. 2012;12:5.

149. Jungquist CR, Tra Y, Smith MT, Pigeon WR, Matteson-Rusby S, Xia Y, et al. The durability of cognitive behavioral therapy for insomnia in patients with chronic pain. Sleep Disord Print. 2012;2012:679648.

150. Kaldo V, Jernelov S, Blom K, Ljotsson B, Brodin M, Jorgensen M, et al. Guided internet cognitive behavioral therapy for insomnia compared to a control treatment - A randomized trial. Behav Res Ther. 2015;71:90-100. 
Does improving sleep lead to better mental health?

151. Kalmbach DA, Cheng P, Arnedt JT, Anderson JR, Roth T, Fellman-Couture C, et al. Treating insomnia improves depression, maladaptive thinking, and hyperarousal in postmenopausal women: comparing cognitive-behavioral therapy for insomnia (CBTI), sleep restriction therapy, and sleep hygiene education. Vol. 55, Sleep medicine. 2019. p. 124-134.

152. Katofsky I, Backhaus J, Junghanns K, Rumpf HJ, Huppe M, von Eitzen U, et al. Effectiveness of a cognitive behavioral self-help program for patients with primary insomnia in general practice - a pilot study. Sleep Med. 2012;13(5):463-8.

153. Kyle SD, Hurry MED, Emsley R, Marsden A, Omlin X, Juss A, et al. The effects of digital cognitive behavioral therapy for insomnia on cognitive function: A randomized controlled trial. Vol. 43, Sleep. 2020. p. 1-12.

154. Lancee J, van den Bout J, van Straten A, Spoormaker VI. Internet-delivered or mailed selfhelp treatment for insomnia?: a randomized waiting-list controlled trial. Behav Res Ther. 2012 Jan;50(1):22-9.

155. Lancee J, van den Bout J, Sorbi MJ, van Straten A. Motivational support provided via email improves the effectiveness of internet-delivered self-help treatment for insomnia: a randomized trial. Behav Res Ther. 2013;51(12):797-805.

156. Lee B, Kim BK, Kim HJ, Jung IC, Kim AR, Park HJ, et al. Efficacy and Safety of Electroacupuncture for Insomnia Disorder: A Multicenter, Randomized, Assessor-Blinded, Controlled Trial. Vol. 12, Nature \& Science of Sleep. 2020. p. 1145-59.

157. Lichstein KL, Nau SD, Wilson NM, Aguillard RN, Lester KW, Bush AJ, et al. Psychological treatment of hypnotic-dependent insomnia in a primarily older adult sample. Behav Res Ther. 2013;51(12):787-96.

158. Martinez MP, Miro E, Sanchez AI, Diaz-Piedra C, Caliz R, Vlaeyen JW, et al. Cognitivebehavioral therapy for insomnia and sleep hygiene in fibromyalgia: a randomized controlled trial. J Behav Med. 2014;37(4):683-97.

159. McCrae CS, Williams J, Roditi D, Anderson R, Mundt JM, Miller MB, et al. Cognitive behavioral treatments for insomnia and pain in adults with comorbid chronic insomnia and fibromyalgia: clinical outcomes from the SPIN randomized controlled trial. Sleep. 2019; $42(3)$.

160. McCurry SM, Logsdon RG, Vitiello MV, Teri L. Successful behavioral treatment for reported sleep problems in elderly caregivers of dementia patients: a controlled study. J Gerontol Ser B-Psychol Sci Soc Sci. 1998;53(2):P122-129.

161. Nguyen S, McKay A, Wong D, Rajaratnam SM, Spitz G, Williams G, et al. Cognitive Behavior Therapy to Treat Sleep Disturbance and Fatigue After Traumatic Brain Injury: A Pilot Randomized Controlled Trial. Arch Phys Med Rehabil. 2017;98(8):1508-1517.e1502. 
Does improving sleep lead to better mental health?

162. Nguyen S, Wong D, McKay A, Rajaratnam SMW, Spitz G, Williams G, et al. Cognitive behavioural therapy for post-stroke fatigue and sleep disturbance: a pilot randomised controlled trial with blind assessment. Neuropsychol Rehabil. 2019;29(5):723-38.

163. Norell-Clarke A, Jansson-Frojmark M, Tillfors M, Hollandare F, Engstrom I. Group cognitive behavioural therapy for insomnia: Effects on sleep and depressive symptomatology in a sample with comorbidity. Behav Res Ther. 2015;74:80-93.

164. Park SD, Yu SH. The effects of Nordic and general walking on depression disorder patients' depression, sleep, and body composition. J Phys Ther Sci. 2015;27(8):2481-5.

165. Peoples AR, Garland SN, Pigeon WR, Perlis ML, Wolf JR, Heffner KL, et al. Cognitive behavioral therapy for insomnia reduces depression in cancer survivors. J Clin Sleep Med. 2019;15(1):129-137.

166. Raskind MA, Peterson K, Williams T, Hoff DJ, Hart K, Holmes H, et al. A trial of prazosin for combat trauma PTSD with nightmares in active-duty soldiers returned from Iraq and Afghanistan. Am J Psychiatry. 2013;170(9):1003-10.

167. Sadler P, McLaren S, Klein B, Harvey J, Jenkins M. Cognitive behavior therapy for older adults with insomnia and depression: A randomized controlled trial in community mental health services. Sleep. 2018;41(8).

168. Sato D, Yoshinaga N, Nagai E, Nagai K, Shimizu E. Effectiveness of Internet-Delivered Computerized Cognitive Behavioral Therapy for Patients With Insomnia Who Remain Symptomatic Following Pharmacotherapy: Randomized Controlled Exploratory Trial. J Med Internet Res. 2019;21(4):e12686.

169. Savard J, Simard S, Ivers H, Morin CM. Randomized study on the efficacy of cognitivebehavioral therapy for insomnia secondary to breast cancer, part I: Sleep and psychological effects. J Clin Oncol. 2005;23(25):6083-96.

170. Schiller H, Soderstrom M, Lekander M, Rajaleid K, Kecklund G. A randomized controlled intervention of workplace-based group cognitive behavioral therapy for insomnia. Int Arch Occup Environ Health. 2018;91(4):413-24.

171. Sheaves B, Freeman D, Isham L, McInerney J, Nickless A, Yu LM, et al. Stabilising sleep for patients admitted at acute crisis to a psychiatric hospital (OWLS): an assessor-blind pilot randomised controlled trial. Psychol Med. 2017;1-11.

172. Sheaves B, Holmes EA, Rek S, Taylor KM, Nickless A, Waite F, et al. Cognitive Behavioural Therapy for Nightmares for Patients with Persecutory Delusions (Nites): an Assessor-Blind, Pilot Randomized Controlled Trial. Vol. 64, Canadian journal of psychiatry. Revue canadienne de psychiatrie. 2019. p. 686-696.

173. Song ML, Park KM, Motamedi GK, Cho YW. Cognitive behavioral therapy for insomnia in restless legs syndrome patients. Vol. 74, Sleep Medicine. 2020. p. 227-34. 
174. Tek C, Palmese LB, Krystal AD, Srihari VH, DeGeorge PC, Reutenauer EL, et al. The impact of eszopiclone on sleep and cognition in patients with schizophrenia and insomnia: a double-blind, randomized, placebo-controlled trial. Schizophr Res. 2014;160(1-3):180-5.

175. Thiart H, Lehr D, Ebert DD, Berking M, Riper H. Log in and breathe out: internet-based recovery training for sleepless employees with work-related strain - results of a randomized controlled trial. Scand J Work Environ Health. 2015;41(2):164-74.

176. Wagley J. Efficacy of a brief intervention for insomnia among psychiatric outpatients. Diss Abstr Int Sect B Sci Eng. 2010;71(1-B):676.

177. Wen X, Wu Q, Liu J, Xu Z, Fan L, Chen X, et al. Randomized single-blind multicenter trial comparing the effects of standard and augmented acupuncture protocols on sleep quality and depressive symptoms in patients with depression. Psychol Health Med. 2018;23(4):375-390.

178. Yeung WF, Chung KF, Tso KC, Zhang SP, Zhang ZJ, Ho LM. Electroacupuncture for residual insomnia associated with major depressive disorder: a randomized controlled trial. Sleep. 2011;34(6):807-15.

179. Zhang L, Tang Y, Hui R, Zheng H, Deng Y, Shi Y, et al. The effects of active acupuncture and placebo acupuncture on insomnia patients: a randomized controlled trial. Vol. 25, Psychology Health \& Medicine. 2020. p. 1201-15.

180. Zhu D, Dai G, Xu D, Xu X, Geng J, Zhu W, et al. Long-Term Effects of Tai Chi Intervention on Sleep and Mental Health of Female Individuals With Dependence on Amphetamine-Type Stimulants. Front Psychol. 2018;9:1476. 
Does improving sleep lead to better mental health?

\section{Table 1}

Search Terms Used to Identify RCT's that Examined the Effect of Improving Sleep on Mental

\section{Health}

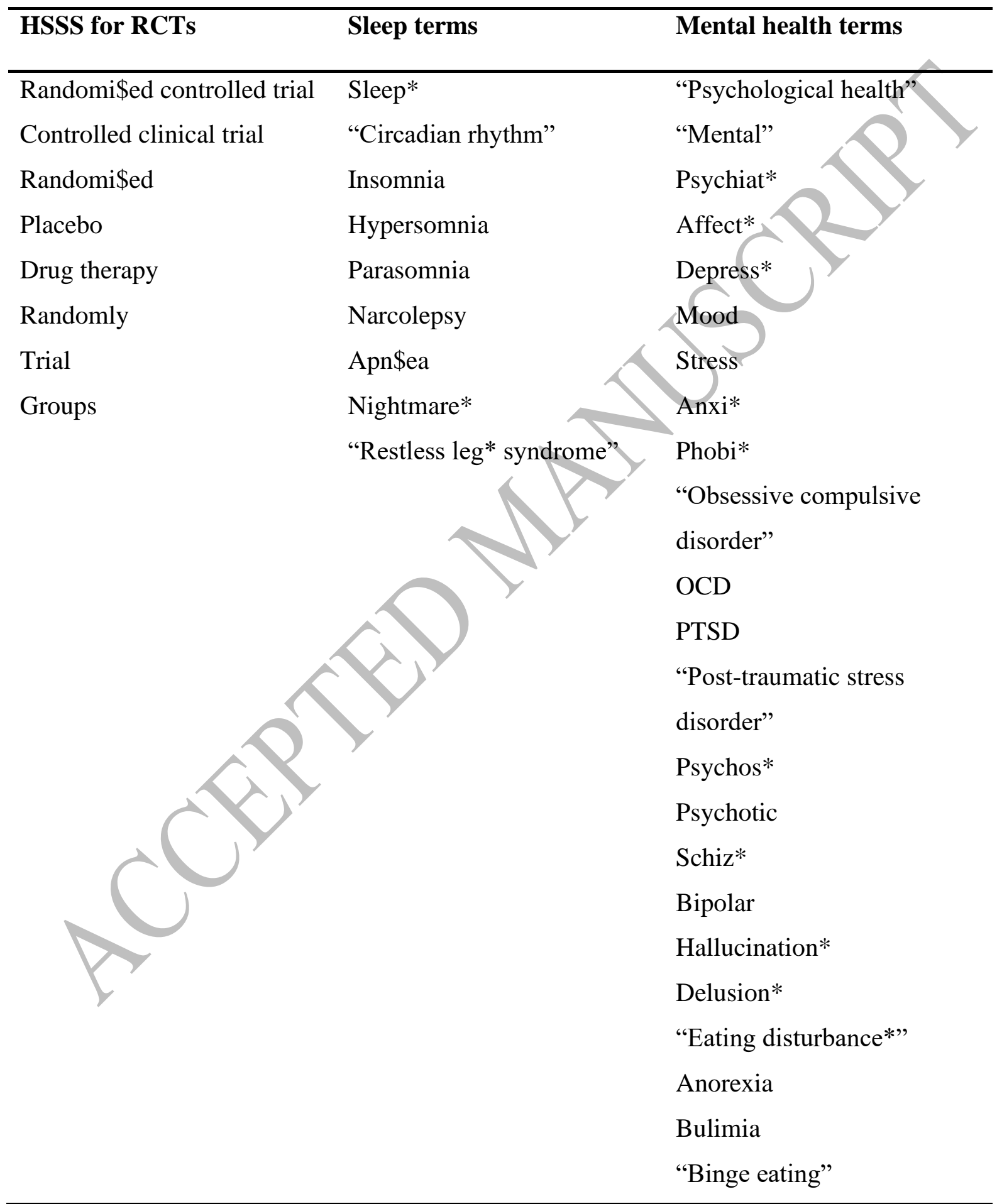


Does improving sleep lead to better mental health?

Notes: HSSS for RCTs = highly sensitive search strategy for randomised controlled trials, OCD $=$ obsessive compulsive disorder, PTSD $=$ post-traumatic stress disorder

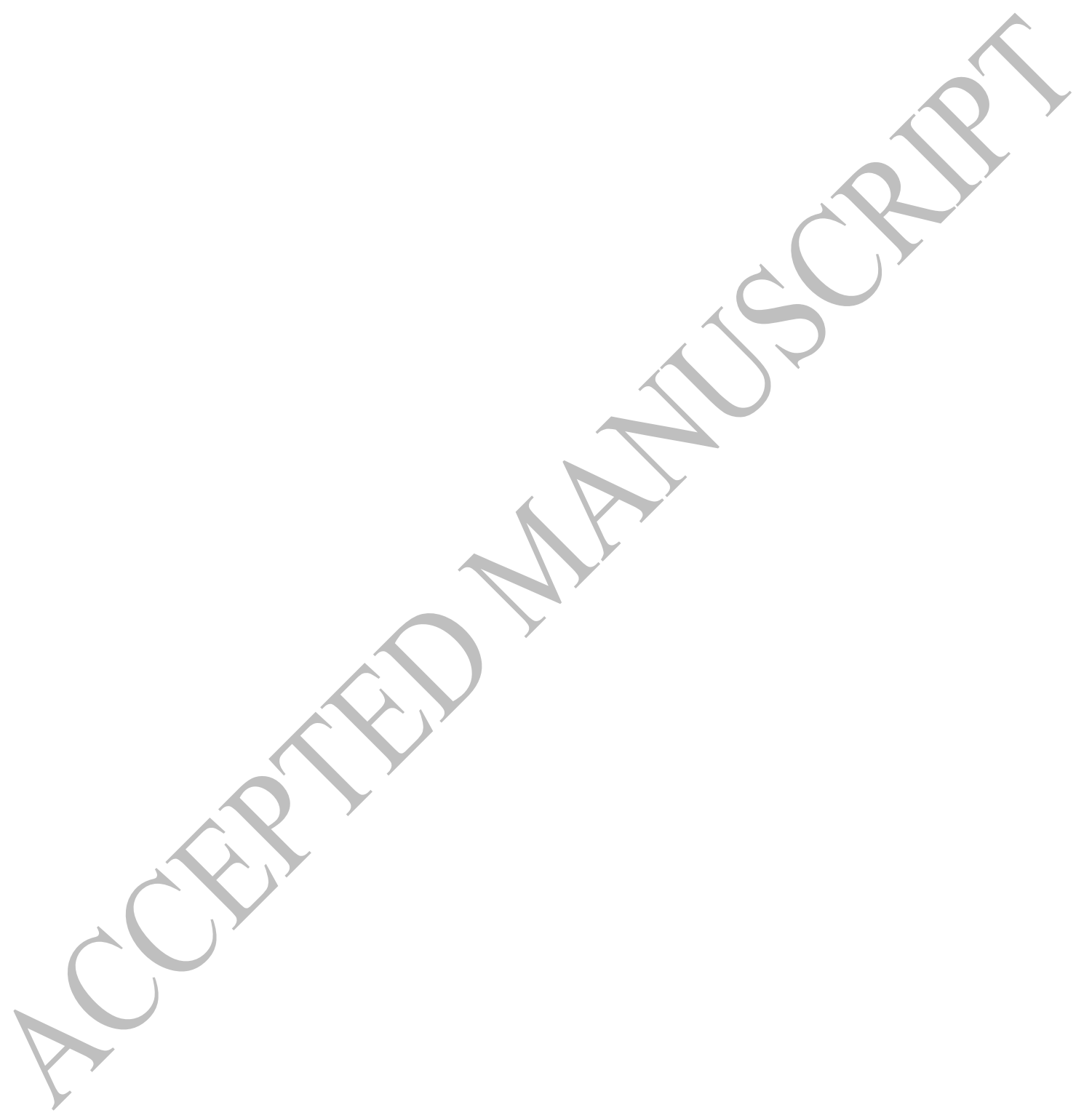


Does improving sleep lead to better mental health?

\section{Table 2}

Summary of Studies Included in the Review

\begin{tabular}{|c|c|c|c|c|c|c|c|}
\hline Author (year) & Intervention & Control & Outcome & Measure & $n_{e}$ & $\overline{n_{c}}$ & $\overline{g_{+}}$ \\
\hline Alessi et al. (2016) (117) & CBTi & Sleep education & Depression & PHQ-9 & 89 & 51 & 0.20 \\
\hline \multirow{2}{*}{$\begin{array}{l}\text { Ashworth et al. (2015) } \\
\text { (118) }\end{array}$} & CBTi & CBTi (self-help) & Anxiety & DASS-A & 18 & 18 & $-1.41 * * *$ \\
\hline & & & Depression & BDI & 18 & 18 & $-2.31 * * *$ \\
\hline \multirow{2}{*}{$\begin{array}{l}\text { Behrendt et al. (2020) } \\
\text { (119) }\end{array}$} & CBTi & WLC & Depression & CES-D & 46 & 80 & $-0.52 * *$ \\
\hline & & & Rumination & PSWQ & 46 & 80 & $-0.45^{*}$ \\
\hline \multirow{2}{*}{$\begin{array}{l}\text { Bergdahl et al. (2016) } \\
(120)\end{array}$} & CBTi & Acupuncture & Anxiety & HADS-A & 23 & 22 & 0.03 \\
\hline & & & Depression & HADS-D & 23 & 22 & 0.06 \\
\hline Blom et al. (2017) (121) & CBTi & ${ }^{2}$ & Depression & MADRS & 20 & 17 & -0.31 \\
\hline \multirow[t]{2}{*}{ Cape et al. (2016) (122) } & CBTi & $\mathrm{TaU}$ & Anxiety & GAD-7 & 91 & 99 & -0.11 \\
\hline & & & Depression & PHQ-9 & 92 & 100 & -0.20 \\
\hline \multirow[t]{2}{*}{ Casault et al. (2015) (123) } & CB & WLC & Anxiety & HADS-A & 17 & 18 & -0.39 \\
\hline & & & Depression & HADS-D & 17 & 18 & -0.11 \\
\hline Chang et al. (2016) (124) & Herbal tea & WLC & Depression & EPDS & 35 & 37 & $-0.52 *$ \\
\hline Chang et al. (2016) (125) & $\begin{array}{l}\text { Sleep education }+ \\
\text { relaxation }\end{array}$ & WLC & Anxiety & HADS-A & 43 & 41 & $-0.68 * *$ \\
\hline
\end{tabular}




\begin{tabular}{|c|c|c|c|c|c|c|c|}
\hline & & & Depression & HADS-D & 43 & 41 & $-0.52 *$ \\
\hline \multirow[t]{2}{*}{ Chao et al. (2021) (126) } & CBTi & WLC & Depression & HADS-D & 32 & 39 & $-0.67 * *$ \\
\hline & & & Anxiety & HADS-A & 32 & 39 & $-0.60 *$ \\
\hline Chen et al. (2009) (127) & Yoga & $\mathrm{TaU}$ & Depression & & 62 & 66 & $-0.60 * * *$ \\
\hline Chen et al. (2019) (128) & Acupuncture & Sham acupuncture & Mood/affect & $\mathrm{K}-10$ & 31 & 31 & -0.50 \\
\hline Cheng et al. (2019) (129) & CBTi & Sleep education & Depression & QIDS & 358 & 300 & $-0.45 * * *$ \\
\hline $\begin{array}{l}\text { Christensen et al. (2016) } \\
\text { (130) }\end{array}$ & \multicolumn{7}{|c|}{$(130)$} \\
\hline Chung et al. (2018) (131) & Acupuncture & \multirow[t]{2}{*}{ WLC } & $\begin{array}{l}\text { Depression } \\
\text { Anxiety } \\
\text { Depression }\end{array}$ & $\begin{array}{l}\text { PHQ-9 } \\
\text { HADS-A } \\
\text { HADS-D }\end{array}$ & $\begin{array}{l}224 \\
71 \\
71\end{array}$ & $\begin{array}{l}280 \\
32 \\
32\end{array}$ & $\begin{array}{l}-0.53 * * * \\
-0.37 \\
-0.46^{*}\end{array}$ \\
\hline Currie et al. (2000) (132) & CBTi & & Depression & BDI & 32 & 28 & -0.31 \\
\hline $\begin{array}{l}\text { Edinger et al. (2005) }{ }^{\mathrm{a}} \\
(133)\end{array}$ & CBTi & & Mood/affect & POMS & 6 & 7 & -1.27 \\
\hline $\begin{array}{l}\text { Edinger et al. }(2005)^{b} \\
(133)\end{array}$ & Sleep hygiene & $\mathrm{TaU}$ & Mood/affect & POMS & 7 & 7 & -1.00 \\
\hline \multirow[t]{2}{*}{ Espie et al. (2008) (81) } & \multirow{5}{*}{ CBTi } & \multirow[t]{2}{*}{ Sleep hygiene } & Anxiety & HADS-A & 67 & 39 & $-0.52 *$ \\
\hline & & & Depression & HADS-D & 67 & 39 & $-0.59 * *$ \\
\hline \multirow[t]{3}{*}{ Espie et al. (2014) (134) } & & $\mathrm{TaU}$ & Anxiety & DASS-A & 40 & 47 & $-0.79 * * *$ \\
\hline & & & Depression & DASS-D & 40 & 47 & $-0.94 * * *$ \\
\hline & & & Stress & DASS-S & 40 & 47 & $-0.93 * * *$ \\
\hline Espie et al. (2019) (135) & CBTi & WLC & Anxiety & GAD-7 & 411 & 495 & $-0.31 * * *$ \\
\hline
\end{tabular}




\begin{tabular}{|c|c|c|c|c|c|c|c|}
\hline & & & Depression & PHQ-9 & 411 & 495 & $-0.39 * * *$ \\
\hline \multirow[t]{2}{*}{ Falloon et al. (2015) (136) } & Sleep restriction & Sleep hygiene & Anxiety & GAD-7 & 43 & 50 & $-0.50 *$ \\
\hline & & & Depression & PHQ-9 & 43 & 50 & -0.27 \\
\hline \multirow[t]{2}{*}{ Felder et al. (2020) (137) } & CBTi & $\mathrm{TaU}$ & Depression & & 88 & 91 & $-0.40 * *$ \\
\hline & & & Anxiety & & 88 & 90 & $-0.37 *$ \\
\hline \multirow{4}{*}{$\begin{array}{l}\text { Freeman et al. (2015) } \\
\text { (138) }\end{array}$} & CBTi & $\mathrm{TaU}$ & Delusions & PSYRATS & 23 & 25 & -0.24 \\
\hline & & & Hallucinations & PSYRATS & 23 & 25 & -0.23 \\
\hline & & & Paranoia & GPTS & 20 & 25 & -0.28 \\
\hline & & & Psychosis & PANSS tot & 21 & 24 & -0.07 \\
\hline \multirow{4}{*}{$\begin{array}{l}\text { Freeman et al. (2017) } \\
\text { (139) }\end{array}$} & CBTi & $\mathrm{TaU}$ & Anxiety & GAD-7 & 603 & 971 & $-0.26 * * *$ \\
\hline & & & Depression & PHQ-9 & 603 & 971 & $-0.35 * * *$ \\
\hline & & & Hallucinations & SPEQ & 603 & 971 & $-0.27 * * *$ \\
\hline & & & Paranoia & GPTS & 603 & 971 & $-0.27 * * *$ \\
\hline \multirow[t]{2}{*}{ Garland et al. (2014) (140) } & CBTi & Mindfulness & Mood/affect & POMS & 40 & 32 & -0.19 \\
\hline & & & Stress & C-SOSI & 40 & 32 & -0.26 \\
\hline \multirow[t]{2}{*}{ Garland et al. (2019) (141) } & 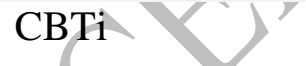 & Acupuncture & Anxiety & HADS-A & 73 & 75 & 0.02 \\
\hline & & & Depression & HADS-D & 73 & 75 & -0.09 \\
\hline \multirow[t]{3}{*}{ Germain et al (2012) (142) } & $\mathrm{CBTi}+\mathrm{IRT}$ & Prazosin placebo & Anxiety & BAI & 12 & 12 & -0.28 \\
\hline & & & Depression & BDI & 12 & 12 & -0.36 \\
\hline & & & PTSD & PCL & 12 & 12 & -0.46 \\
\hline
\end{tabular}




\begin{tabular}{|c|c|c|c|c|c|c|c|}
\hline Glozier et al. (2019) (143) & CBTi & Sleep education & Depression & CES-D & 31 & 28 & -0.03 \\
\hline Ham et al. (2020) (144) & CBTi & Sleep hygiene & Depression & CES-D & 24 & 20 & -0.56 \\
\hline \multirow[t]{2}{*}{ Ho et al. (2014) ${ }^{\mathrm{a}}(145)$} & $\mathrm{CBTi}+$ telephone & WLC & Anxiety & HADS-A & 49 & 33 & -0.21 \\
\hline & & & Depression & & 49 & 33 & -0.13 \\
\hline \multirow[t]{2}{*}{ Ho et al. (2014) ${ }^{b}(145)$} & CBTi & WLC & Anxiety & HADS-A & 45 & 33 & -0.19 \\
\hline & & & Depression & HADS-D & 45 & 33 & -0.16 \\
\hline Irwin et al. (2014) ${ }^{\mathrm{a}}(146)$ & CBTi & WLC & Depression & IDS-C & 46 & 11 & -0.63 \\
\hline Irwin et al. (2014) ${ }^{\mathrm{b}}(146)$ & Tai Chi & WLC & Depression & IDS-C & 39 & 12 & -0.22 \\
\hline \multirow{2}{*}{$\begin{array}{l}\text { Jansson-Frojmark et al. } \\
(2012)(147)\end{array}$} & CBTi & WLC & & HADS-A & 15 & 15 & $-1.19 * *$ \\
\hline & & & Depression & HADS-D & 15 & 15 & $-1.12 * *$ \\
\hline \multirow{3}{*}{$\begin{array}{l}\text { Jernelov et al. }(2012)^{a} \\
(148)\end{array}$} & CBTi + telephone & WLC & Mood/affect & CORE-OM & 44 & 22 & -0.50 \\
\hline & support & & & & & & \\
\hline & & & Stress & PSS & 44 & 22 & $-0.64 *$ \\
\hline \multirow{2}{*}{$\begin{array}{l}\text { Jernelov et al. }(2012)^{b} \\
(148)\end{array}$} & CBTi & & Mood/affect & CORE-OM & 45 & 22 & -0.39 \\
\hline & & & Stress & PSS & 45 & 22 & -0.30 \\
\hline $\begin{array}{l}\text { Jungquist et al. (2012) } \\
\text { (149) }\end{array}$ & CBTi & Self-monitoring & Depression & BDI & 14 & 4 & $-2.44 * * *$ \\
\hline $\begin{array}{l}\text { Kaldo, V et al. (2015) } \\
(150)\end{array}$ & CBTi & $\begin{array}{l}\text { Mindfulness + sleep } \\
\text { hygiene + relaxation }\end{array}$ & Stress & PSS & 54 & 53 & 0.00 \\
\hline
\end{tabular}




\begin{tabular}{|c|c|c|c|c|c|c|c|}
\hline \multirow{3}{*}{$\begin{array}{l}\text { Kalmbach et al. (2019) } \\
\text { (151) }\end{array}$} & \multirow[t]{3}{*}{ CBTi } & \multirow{3}{*}{ Sleep hygiene } & Depression & BDI-II & 42 & 20 & -0.45 \\
\hline & & & Rumination & ERRI & 42 & 20 & -0.17 \\
\hline & & & Rumination & PSWQ & 42 & 20 & -0.38 \\
\hline \multirow{3}{*}{$\begin{array}{l}\text { Kalmbach et al. (2019) } \\
\text { (151) }\end{array}$} & \multirow[t]{3}{*}{ CBTi } & \multirow[t]{3}{*}{ Sleep hygiene } & Depr & BDI-II & 34 & 20 & -0.51 \\
\hline & & & & ERRI & 34 & 20 & -0.08 \\
\hline & & & & PSWQ & 34 & 20 & -0.53 \\
\hline $\begin{array}{l}\text { Katofsky et al. (2012) } \\
\text { (152) }\end{array}$ & $\begin{array}{l}\text { CBTi + sleep } \\
\text { medication }\end{array}$ & Sleep medication & Depression & BDI & 41 & 39 & -0.11 \\
\hline \multirow[t]{2}{*}{ Kyle et al. (2020) (153) } & \multirow[t]{2}{*}{ CBTi } & \multirow[t]{2}{*}{ WLC } & Depression & PHQ-9 & 136 & 166 & $-0.53 * * *$ \\
\hline & & & Anxiety & GAD-7 & 136 & 166 & $-0.33 * *$ \\
\hline \multirow[t]{2}{*}{ Lancee et al. (2012) ${ }^{\mathrm{a}}(154)$} & \multirow[t]{2}{*}{ CBTi (digital) } & \multirow[t]{2}{*}{ WLC } & Anxiety & HADS-A & 109 & 92 & -0.17 \\
\hline & & & Depression & CES-D & 109 & 42 & -0.23 \\
\hline \multirow[t]{2}{*}{ Lancee et al. (2012) ${ }^{\mathrm{b}}(154)$} & \multirow[t]{2}{*}{ CBTi (booklet) } & \multirow[t]{2}{*}{ WLC } & Anxiety & HADS-A & 126 & 91 & -0.02 \\
\hline & & & Depression & CES-D & 126 & 41 & -0.03 \\
\hline \multirow[t]{2}{*}{ Lancee et al. (2013) (155) } & \multirow[t]{2}{*}{ CBTi } & \multirow[t]{2}{*}{ CBTi (self-help) } & Anxiety & HADS-A & 102 & 95 & -0.16 \\
\hline & & & Depression & CES-D & 102 & 95 & $-0.32 *$ \\
\hline \multirow[t]{2}{*}{ Lee et al. (2020) (156) } & \multirow{2}{*}{ Acupuncture } & \multirow[t]{2}{*}{ WLC } & Depression & HADS-D & 49 & 49 & $-2.66 * * *$ \\
\hline & & & Anxiety & HADS-A & 49 & 49 & $-0.91 * * *$ \\
\hline $\begin{array}{l}\text { Lichstein et al. (2013) } \\
\text { (157) }\end{array}$ & CBTi & Hypnotic taper & Anxiety & STAI & 22 & 18 & -0.35 \\
\hline
\end{tabular}




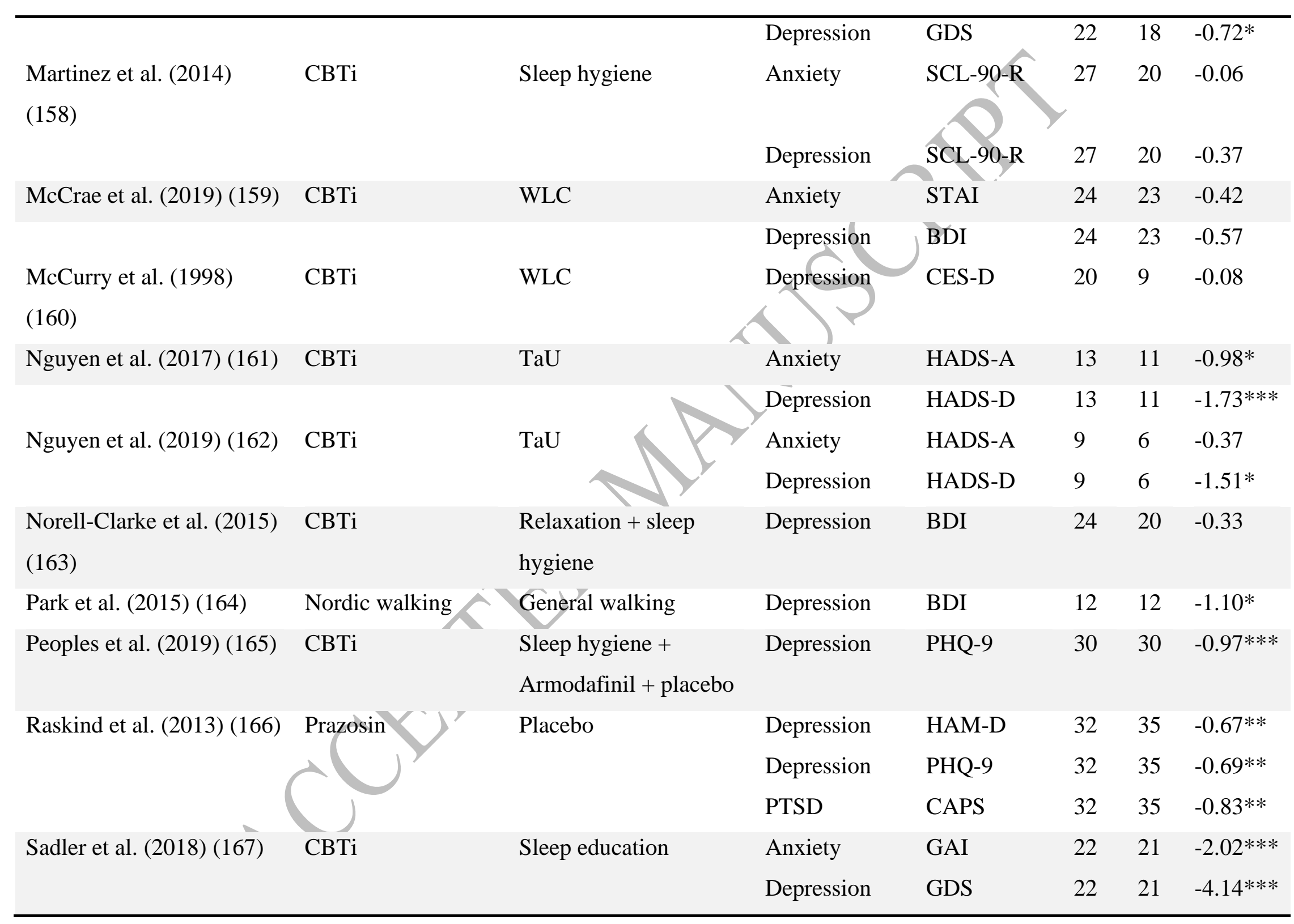


Does improving sleep lead to better mental health?

\begin{tabular}{|c|c|c|c|c|c|c|c|}
\hline \multirow[t]{2}{*}{ Sato et al. (2019) (168) } & \multirow{2}{*}{ CBTi } & \multirow[t]{2}{*}{$\mathrm{TaU}$} & Anxiety & HADS-A & 11 & 11 & -0.81 \\
\hline & & & Depression & CES-D & 11 & 11 & $-1.52 * *$ \\
\hline \multirow[t]{2}{*}{ Savard et al. (2005) (169) } & \multirow[t]{2}{*}{ CBTi } & \multirow[t]{2}{*}{ WLC } & Anxiety & HADS-A & 27 & 30 & 0.35 \\
\hline & & & Depression & HADS-D & 27 & 30 & 0.27 \\
\hline Schiller et al. (2018) (170) & CBTi & WLC & Burnout & SMBQ & 25 & 26 & -0.03 \\
\hline \multirow[t]{5}{*}{ Sheaves et al. (2017) (171) } & \multirow[t]{5}{*}{ CBTi } & \multirow[t]{5}{*}{$\mathrm{TaU}$} & Suicidal & BSS & 20 & 20 & -0.14 \\
\hline & & & ideation & & & & \\
\hline & & & Psychosis & PANSS pos & 20 & 20 & -0.31 \\
\hline & & & Psychosis & PANSS neg & 20 & 20 & -0.51 \\
\hline & & & Psychosis & PANSS tot & 20 & 20 & -0.34 \\
\hline \multirow[t]{9}{*}{ Sheaves et al. (2019) (172) } & \multirow[t]{9}{*}{ CBT for nightmares } & \multirow[t]{9}{*}{$\mathrm{TaU}$} & Anxiety & DASS-A & 11 & 9 & -0.65 \\
\hline & & & Depression & DASS-D & 11 & 9 & 0.15 \\
\hline & & & Dissociation & DES-B & 11 & 9 & -0.73 \\
\hline & & & Hallucinations & CAPS & 11 & 9 & -0.10 \\
\hline & & & Paranoia & GPTS & 11 & 9 & -0.82 \\
\hline & & & Psychosis & DES-B & 11 & 9 & -0.73 \\
\hline & & & Stress & DASS-S & 11 & 9 & -0.46 \\
\hline & & & Suicidal & BSS & 11 & 9 & 0.48 \\
\hline & & & ideation & & & & \\
\hline \multirow[t]{2}{*}{ Song et al. (2020) (173) } & \multirow[t]{2}{*}{ CBTi } & \multirow[t]{2}{*}{ Sleep hygiene } & Depression & BDI & 12 & 13 & -0.07 \\
\hline & & & Anxiety & BAI & 12 & 13 & $-0.98 *$ \\
\hline Tek et al. (2014) (174) & Eszopiclone & Placebo & Depression & CDS & 19 & 17 & -0.07 \\
\hline
\end{tabular}




\begin{tabular}{|c|c|c|c|c|c|c|c|}
\hline & & & Psychosis & PANSS-pos & 19 & 17 & -0.32 \\
\hline & & & Psychosis & PANSS-neg & 19 & 17 & -0.05 \\
\hline & & & Psychosis & PANSS-tot & 19 & 17 & -0.10 \\
\hline Thiart et al. (2015) (175) & CBTi & WLC & Rumination & PSWQ & 59 & 54 & $-0.84 * * *$ \\
\hline Wagley (2010) (176) & CBTi & WLC & Depression & PHQ-9 & 24 & 10 & $-1.55 * * *$ \\
\hline Wen et al. (2018) (177) & $\begin{array}{l}\text { Augmented } \\
\text { acupuncture }\end{array}$ & Standard acupuncture & Depression & HADS-D & 43 & 46 & $-1.01 * * *$ \\
\hline Yeung et al. $(2011)^{\mathrm{a}}(178)$ & Electroacupuncture & Placebo acupuncture & Depression & HDRS & 22 & 11 & -0.28 \\
\hline Yeung et al. $(2011)^{\mathrm{b}}(178)$ & $\begin{array}{l}\text { Standard } \\
\text { acupuncture }\end{array}$ & Placebo acupuncture & Depression & HDRS & 23 & 12 & -0.47 \\
\hline Zhang et al. (2020) (179) & Acupuncture & Sham acupunctur & $\begin{array}{l}\text { Depression } \\
\text { Anxiety }\end{array}$ & $\begin{array}{l}\text { SDS } \\
\text { SAS }\end{array}$ & $\begin{array}{l}46 \\
46\end{array}$ & $\begin{array}{l}44 \\
44\end{array}$ & $\begin{array}{l}-3.56 * * * \\
-3.93 * * *\end{array}$ \\
\hline Zhu et al. (2018) (180) & Tai Chi & $\mathrm{TaU}$ & Depression & SDS & 37 & 12 & -0.30 \\
\hline
\end{tabular}

Note: $* p<0.05, * * p<0.01, * * * p<0.001$. CBTi = cognitive behavioural therapy for insomnia, $\mathrm{dx}=$ diagnosis, IRT $=$ image rehearsal therapy, $\mathrm{MH}=$ mental health, $n_{\mathrm{e}}=$ number of participants in intervention group, $n_{\mathrm{c}}=$ number of participants in the control group,

PTSD = post-traumatic stress disorder, TaU = treatment as usual, WLC $=$ wait list control. ${ }^{\text {ab }}$ Subscript indicates that the study reports multiple eligible interventions in the same study, in these situations both interventions were included as separate studies in the analysis and the control was halved accordingly. 
Does improving sleep lead to better mental health?

\section{$1 \quad$ Table 3}

2 The Effect of Improving Sleep on Mental Health Outcomes

\begin{tabular}{|c|c|c|c|c|c|c|}
\hline Outcome & $\overline{g_{+}}$ & $95 \% \mathrm{CI}$ & $I^{2}$ & $Q$ & $\boldsymbol{k}$ & $\bar{N}$ \\
\hline Composite outcomes & $-0.53 * * *$ & -0.69 to -0.38 & $76 \%$ & $291.94 * * *$ & 72 & 8608 \\
\hline Depression & $-0.63 * * *$ & -0.84 to -0.43 & $81 \%$ & $322.03 * * *$ & 61 & 7868 \\
\hline Anxiety & $-0.51 * * *$ & -0.77 to -0.24 & $82 \%$ & $186.92 * * *$ & & 5819 \\
\hline Stress & $-0.42 *$ & -0.79 to -0.05 & $55 \%$ & 11.05 & & 419 \\
\hline \multicolumn{7}{|l|}{ Psychosis spectrum } \\
\hline PANSS total & -0.17 & -0.53 to 0.19 & 7 & 0.41 & 3 & 121 \\
\hline Positive symptoms & $-0.26^{*}$ & -0.43 to -0.08 & & 71 & 5 & 1715 \\
\hline Negative & -0.28 & -3 & & 1.00 & 2 & 76 \\
\hline \multicolumn{7}{|l|}{ symptoms } \\
\hline Suicidal ideation & 0.10 & 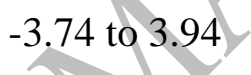 & $20 \%$ & 1.25 & 2 & 60 \\
\hline PTSD & -0.72 & 0.46 & $0 \%$ & 0.59 & 2 & 91 \\
\hline Rumination & -0 & -0.93 to -0.04 & $36 \%$ & 4.65 & 4 & 355 \\
\hline Burnout & & -0.58 to 0.52 & - & - & 1 & 51 \\
\hline
\end{tabular}

4 Post Traumatic Stress Disorder. 
Does improving sleep lead to better mental health?

\section{Table 4}

6 Categorical Moderators of the Effect of Improving Sleep on Composite Mental Health

$7 \quad$ Outcomes

\begin{tabular}{|c|c|c|c|c|c|}
\hline Variable & Levels & $\boldsymbol{k}$ & $g_{+}$ & $95 \% \mathrm{CI}$ & $Q$ \\
\hline \multirow{3}{*}{$\begin{array}{l}\text { Significant effect on } \\
\text { sleep }^{\text {a }}\end{array}$} & Yes & 72 & -0.53 & -0.69 to - & $17.69 * * *$ \\
\hline & & & & 0.38 & \\
\hline & No & 31 & -0.12 & -0.24 to 0.01 & \\
\hline \multirow[t]{2}{*}{ Clinical status of $\mathrm{MH}$} & Clinical & 15 & -0.72 & -1.14 tc & 0.92 \\
\hline & Non-clinical & 45 & -0.50 & & \\
\hline \multirow[t]{5}{*}{ Comorbidities } & Mental health & 18 & & -1.00 to - & 0.63 \\
\hline & Physical health & & & -0.76 to - & \\
\hline & & & & 0.32 & \\
\hline & No comorb & 31 & -0.47 & -0.72 to - & \\
\hline & & & & 0.23 & \\
\hline \multirow[t]{4}{*}{ Follow-up point } & Shor & 61 & -0.60 & -0.77 to - & $10.75^{* *}$ \\
\hline & & & & 0.42 & \\
\hline & & 11 & -0.18 & -0.36 to - & \\
\hline & months) & & & 0.00 & \\
\hline \multirow[t]{4}{*}{ Assessment } & Self-reported & 66 & -0.54 & -0.70 to - & 0.62 \\
\hline & & & & 0.38 & \\
\hline & Clinician rated & 6 & -0.44 & -0.65 to - & \\
\hline & & & & 0.23 & \\
\hline \multirow{4}{*}{ Adjusted data } & Adjusted & 21 & -0.51 & -0.77 to - & 0.01 \\
\hline & & & & 0.26 & \\
\hline & Unadjusted & 51 & -0.53 & -0.72 to - & \\
\hline & & & & 0.35 & \\
\hline \multirow[t]{2}{*}{ Recruitment setting } & Clinical (MH) & 12 & -0.52 & -1.00 to - & 3.72 \\
\hline & & & & 0.04 & \\
\hline
\end{tabular}


Does improving sleep lead to better mental health?

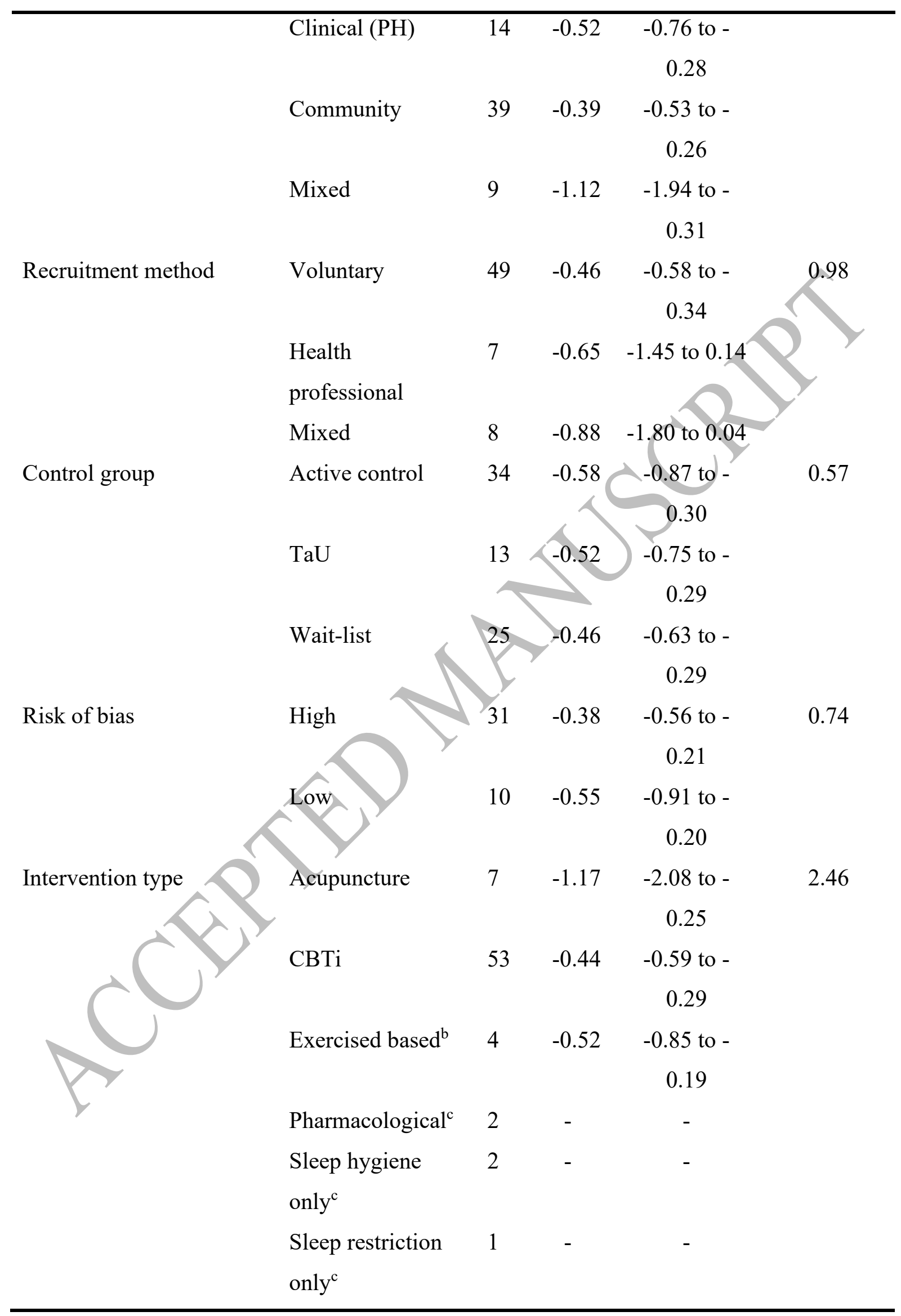


Does improving sleep lead to better mental health?

\begin{tabular}{|c|c|c|c|c|c|}
\hline & CBT for & 1 & - & - & \\
\hline & nightmares $^{\mathrm{c}}$ & & & & \\
\hline & Herbal tea ${ }^{c}$ & 1 & - & - & \\
\hline \multirow[t]{3}{*}{ Intervention format } & Group & 11 & -0.42 & -0.92 to 0.08 & 0.25 \\
\hline & Individual & 52 & -0.55 & -0.73 to - & \\
\hline & & & & 0.38 & \\
\hline \multirow[t]{3}{*}{ Intervention delivery } & Clinician & 43 & -0.63 & -0.87 to - & $.50 *$ \\
\hline & delivered & & & 0.38 & \\
\hline & Self-administered & 23 & -0.34 & -0.43 to & \\
\hline \multirow[t]{18}{*}{ Country of origin } & Australia & 5 & -1.50 & & $53.69 * * *$ \\
\hline & Canada & 4 & & -0.40 to 0.17 & \\
\hline & China & 8 & & -1.59 to - & \\
\hline & Germany & & & -0.90 to - & \\
\hline & & & & 0.08 & \\
\hline & Korea & 4 & -0.78 & -1.70 to 0.15 & \\
\hline & Netherlands & 3 & -0.16 & -0.29 to - & \\
\hline & & & & 0.03 & \\
\hline & & 8 & -0.28 & -0.53 to - & \\
\hline & & & & 0.03 & \\
\hline & Taiwan & 4 & -0.57 & -0.61 to - & \\
\hline & & & & 0.52 & \\
\hline & UK & 9 & -0.36 & -0.51 to - & \\
\hline & & & & 0.22 & \\
\hline & USA & 20 & -0.50 & -0.71 to - & \\
\hline & & & & 0.28 & \\
\hline & New Zealand ${ }^{\mathrm{c}}$ & 2 & - & - & \\
\hline & Spain $^{c}$ & 1 & - & - & \\
\hline
\end{tabular}

8 Notes: $\mathrm{CBTi}=$ cognitive behavioural therapy for insomnia, $\mathrm{MH}=$ Mental Health, $\mathrm{PH}=$

9 Physical Health, TaU $=$ treatment as usual, $\mathrm{WLC}=$ wait list control. Studies in the 'No'

10 moderator category were excluded from the review due to having no significant effect on 
Does improving sleep lead to better mental health?

11 sleep quality but were included for moderation analysis ${ }^{\mathrm{a}}$. The 'exercise based' category

12 combines separate interventions with exercise as a key element, including walking, yoga and

13 Tai $\mathrm{Chi}^{\mathrm{b}}$. Not included in subgroup analysis due to low number ${ }^{\mathrm{c}}{ }^{*} p<0.05,{ }^{* *} p<0.01,{ }^{* * *} p$ $14<0.001$. 
Does improving sleep lead to better mental health?

\section{Table 5}

16 Continuous Moderators of the Effect of Improving Sleep on Composite Mental Health

17 Outcomes

\begin{tabular}{lcccc}
\hline Variable & $\boldsymbol{k}$ & $\mathbf{B}$ & $\mathbf{S E}$ & $\mathbf{9 5 \%}$ CI \\
\hline Publication year & 72 & -0.02 & 0.02 & -0.05 to 0.02 \\
Journal impact & 71 & 0.01 & 0.01 & -0.01 to 0.03 \\
Age & 71 & 0.00 & 0.01 & -0.01 to 0.02 \\
Sex & 71 & 0.00 & 0.00 & -0.00 to 0.01 \\
Sleep effect & 70 & $0.77^{* * *}$ & 0.13 & 0.52 to 1.02 \\
Intervention duration & 70 & 0.02 & 0.02 & -0.03 to 0.06 \\
Contact time & 55 & 0.00 & 0.01 & -0.02 to 0.01 \\
Number of sessions & 61 & 0.00 & 0.01 & -0.02 to 0.01 \\
\hline
\end{tabular}

18 
Does improving sleep lead to better mental health?

19 Figure 1. PRISMA Diagram Showing the Flow of Studies Through the Review

20 Figure 2. Forest Plot Showing the Effect of Improving Sleep on Composite Mental Health

21 Outcomes

22 Figure 3. Forest Plot Showing the Effect of Improving Sleep on Depression

23 Figure 4. Forest Plot Showing the Effect of Improving Sleep on Anxiety

24 Figure 5. Forest Plot Showing the Effect of Improving Sleep on Stress, Suicidal Ideation, 25 PTSD, and rumination

26 Figure 6. Forest Plot Showing the Effect of Improving Sleep on Psychosis Spectrum

27 Outcomes

28 Figure 7. Weighted Risk of Bias Summary Plot

29 Figure 8. Contrast Enhanced Funnel Plot for the Effect of Improving Sleep on Composite

30 Mental Health (Solid Grey Markers) with Imputed Studies (Hollow Markers) 